\title{
No observable guide-RNA-independent off-target mutation induced by prime editor
}

Runze Gao ${ }^{1,2,3,4,6}$, Zhi-Can Fu ${ }^{5,6}$, Xiangyang $\mathrm{Li}^{1,2,3,4,6}$, Ying Wang ${ }^{5,6}$, Jia Wei ${ }^{5}$, Guangye $\mathrm{Li}^{1,2,3,4}$, Lijie Wang ${ }^{1,2,3,4}$, Jing Wu ${ }^{1,2}$, Wei Xue ${ }^{5}$, Xingxu Huang ${ }^{1,2,3 *}$, Li Yang $^{5 *} \&$ Jia Chen ${ }^{1,2,3 *}$

${ }^{1}$ School of Life Science and Technology, ShanghaiTech University, Shanghai 201210, China

${ }^{2}$ Gene Editing Center, School of Life Science and Technology, ShanghaiTech University, Shanghai 201210, China

${ }^{3}$ Shanghai Institute of Biochemistry and Cell Biology, CAS Center for Excellence in Molecular Cell Science, Chinese Academy of Sciences, Shanghai 200031, China ${ }^{4}$ University of Chinese Academy of Sciences, Beijing 100049, China ${ }^{5}$ CAS Key Laboratory of Computational Biology, CAS-MPG Partner Institute for Computational Biology, Shanghai Institute of Nutrition and Health, University of Chinese Academy of Sciences, Chinese Academy of Sciences, Shanghai 200031, China

${ }^{6}$ These authors contributed equally to this work

*Correspondence should be addressed to L.Y. (liyang@picb.ac.cn); X.H. (huangxx@,shanghaitech.edu.cn); J.C. (henjia@,shanghaitech.edu.cn) 


\author{
ABSTRACT \\ Prime editor (PE) has been recently developed to induce efficient and precise on- \\ target editing, whereas its guide RNA (gRNA)-independent off-target effects remain \\ unknown. Here, we used whole-genome and whole-transcriptome sequencing to \\ determine gRNA-independent off-target mutations in cells expanded from single \\ colonies, in which PE generated precise editing at on-target sites. We found that PE \\ triggered no observable gRNA-independent off-target mutation genome-wide or \\ transcriptome-wide in transfected human cells, highlighting its high specificity.
}

\title{
MAIN TEXT
}

Prime editors (PEs) that combine reverse transcriptase (RTase) with CRISPR-Cas9

system have been developed and successfully applied to induce targeted base substitutions, small deletions or insertions in mammalian cells and plants ${ }^{1,2}$. By using the edit-containing genetic information encoded in the reverse transcription (RT) template of prime editing guide RNA (pegRNA), the RTase of PE can incorporate edits into target genomic DNA with high efficiency ${ }^{1,3}$. Distinct to previously reported base editors (BEs), which can generate on-target $\mathrm{C}$-to-T change with the combination of CRISPR-Cas9 and apolipoprotein B mRNA editing enzyme, catalytic polypeptidelike (APOBEC) cytidine deaminase, PEs are versatile to deliver different types of edits. As PE can virtually correct most of mutations associated with genetic disorders 
for therapeutic purposes, whether PEs induce off-target (OT) effects is of great importance for its potential clinic applications ${ }^{4}$. It is well studied that the engineering of Cas9 protein can greatly reduce the binding at OT sites that have sequence similarity to on-target sites $^{4,5}$ to reduce the gRNA-dependent OT mutations. However, whether the effector moiety of PE (RTase) induces gRNA-independent OT mutations, which were recently found to be the major OT effect for $\mathrm{BE}^{6-9}$, remains unknown.

At first, we sought to determine whether PE induces gRNA-independent OT mutations genome-wide. As the family of APOBEC3 cytidine deaminase has been reported to induce mutations in cellular genomic $\mathrm{DNA}^{10-12}$, we knocked out the endogenously expressed $A P O B E C 3$ (A3) gene cluster with CRISPR-Cas9 in 293FT cells (Supplementary Fig. 1a-1d) and obtained a single-clone-derived 293FT ${ }^{A 3-/-}$ cell line, in order to reduce genome-wide mutation background. We next transfected the EGFP-expressing plasmid into wild-type (WT) $293 \mathrm{FT}$ or $293 \mathrm{FT}^{A 3-/-}$ cells, and 72 hours after transfection, single cells were sorted into 96-well plates. After colony expansion, the genomic DNA of single cell colonies was subjected to whole-genome sequencing (WGS) (Fig. 1a). WGS data analyzed by a Base/Prime editor induced DNA off-target site identification unified toolkit (BEIDOU, https://github.com/YangLab/BEIDOU) showed that the knockout of $A 3$ cluster significantly reduced the number of genome-wide base substitutions (Supplementary 
Fig. 2a, from $\sim 4,900$ to $\sim 1,600, P=6 \times 10^{-5}$ ) and insertions or deletions (indels, Supplementary Fig. $2 \mathrm{~b}$, from $\sim 400$ to $\sim 20, P=2 \times 10^{-8}$ ). In the subsequent study, we then used the 293FT ${ }^{A 3-/-}$ cell line with a low mutation background to evaluate the gRNA-independent OT effects induced by PEs genome-wide, with EGFP as the negative control and a previously reported $\mathrm{BE}(\mathrm{hA} 3 \mathrm{~A}-\mathrm{BE} 3)^{13}$ as the positive control for detecting gRNA-independent OT mutations.

We first compared the on-target editing efficiencies of PE3 to hA3A-BE3 with amplicon sequencing in a bulk transfection setting. 293FT ${ }^{A 3-/-}$ cells were transfected with three plasmids expressing PE3, pegRNA (Fig. 1b) and the optimized nicking gRNA $^{1}$ (Supplementary Fig. 3a), two plasmids expressing hA3A-BE3 and gRNA, two plasmids expressing Cas9 and gRNA, or one plasmid expressing EGFP. 72 hours after transfection, a portion of transfected cells was lysed to extract genomic DNA for examining on-target editing frequencies. PE3 generated C-to-T substitutions at $R N F 2$, FANCF and SEC61B target sites, with editing efficiencies similar to those by hA3ABE3 (Fig. 1c). Consistent with previous studies showing that both PE3 and hA3ABE3 triggered indels ${ }^{1,13}$, we found that both editors induced indels at these on-target sites, but fewer than those by Cas9 (Supplementary Fig. 3b). 
Another portion of transfected cells was sorted to single cells and expanded in 96-well plates. After culturing, the single cell colonies with Sanger-sequencing confirmed biallelic edits (100\% editing frequency) at on-target sites (Supplementary Fig. 4a-4c) were subjected to WGS with a depth of at least $12 \times$ to identify OT mutations and analyzed by the BEIDOU toolkit on a genome-wide scale (Supplementary Fig. 5a5d). We found the numbers of base substitutions in PE3-treated (Fig. 1d, $P=0.13$, 0.08 and 0.02, for RNF2, FANCF and SEC61B, respectively) and Cas9-treated single cell colonies (Fig. 1d, $P=0.24,0.02$ and 0.14 , for $R N F 2, F A N C F$ and $S E C 61 B$, respectively) were similar to or slightly smaller than the ones in EGFP-treated single cell colonies, suggesting that PE3 and Cas9 induced no observable genome-wide OT base substitution. In contrast, hA3A-BE3 induced more base substitutions than EGFP control (Fig. 1d, $P=0.004,0.13$ and $4 \times 10^{-5}$, for RNF2, FANCF and SEC61B, respectively), which is in line with previous reports that BE3 induced substantial genome-wide OT mutations in mouse embryos and plants ${ }^{7,9}$. We further analyzed the type of base substitutions and found that significantly more C-to-T or G-to-A substitutions than other subtypes of base substitutions (Fig. 1e and Supplementary Fig. 6a, $P<0.008$ ) were induced by hA3A-BE3, while C-to-T or G-to-A substitutions were not significantly induced by other genome editors (Fig. 1e and Supplementary Fig. 6b). We also analyzed sequence contexts of base substitutions induced by hA3ABE3 and found that the WGS-identified OT mutation sites have little sequence similarity to on-target sites or predicted gRNA-dependent OT sites (Supplementary 
Fig. 7a-7f). This finding is consistent with previous studies $7,9,14,15$ and indicates that these OT mutations are gRNA-independent and randomly induced by the cytidine deaminase moiety of hA3A-BE3 ${ }^{16,17}$. Moreover, we used Sanger sequencing to confirm the WGS-identified gRNA-independent OT mutations at selected loci that are associated with human diseases and found $\sim 50 \%$ mutation frequencies at these pathogenic sites in single cell colonies (Supplementary Fig. 8a and 8b). Other than base substitutions, we also analyzed whether PE3 and hA3A-BE3 induced gRNAindependent OT indels genome-wide when these two editors were used to generate on-target base substitutions. Both of them only manifested background numbers of indels, comparing to Cas9 or EGFP control (Fig. 1f and Supplementary Fig. 9a-9d). These results together demonstrated that PE3 induced no observable gRNAindependent OT base substitution or indel in its application of introducing single base changes.

An advantage of applying PE3 for genome editing is to introduce small deletions or insertions at targeted sites ${ }^{1,2}$. Thus, we further set to evaluate gRNA-independent OT effects in the application of PE3 for generating designed deletions or insertions. With the same strategy shown in Fig. 1a, we transfected 293FT ${ }^{A 3-/-}$ cells with PE3, Cas9 and EGFP. With three pairs of pegRNA and nicking gRNA with optimal editing efficiencies $^{1}$ (Supplementary Fig. 10a), PE3 was used to generate 3-bp deletions at EMX1, HEK1 and LSP1 sites (Fig. 2a) and deep sequencing results showed that PE3 
yielded $\sim 40 \%-70 \%$ intended deletion frequencies in a bulk transfection setting (Fig. 2b). Next, the whole genomes of edited single cell colonies with intended 3-bp deletions (Supplementary Fig. 11a-11c) were sequenced and then analyzed by the BEIDOU toolkit (Supplementary Fig. 12a and 12b). WGS results showed that PE3 induced similar or slightly fewer OT indels, comparing to Cas9 (Fig. 2c, $P=0.32$, 0.15 and 0.06 for $E M X 1, H E K 1$ and $L S P 1$, respectively) or EGFP control (Fig. 2c, $P$ $=0.14,0.11$ and 0.03 for $E M X 1, H E K 1$ and $L S P 1$, respectively). Meanwhile, the numbers of base substitutions induced by PE3 were similar to or slightly smaller than Cas9 (Fig. 2d, $P=0.21,0.23$ and 0.001 for $E M X 1, H E K 1$ and $L S P 1$, respectively) and EGFP control (Fig. 2d, $P=0.07,0.04$ and 0.02 for EMX1, HEK1 and LSP1, respectively), consistent to the results shown in Fig. 1. We further confirmed that these designed 3-bp deletions were not induced at OT sites on a genome-wide scale (Supplementary Fig. 13a-13c).

Finally, we used PE3 and three pairs of pegRNA and nicking gRNA with optimal editing efficiencies ${ }^{1}$ (Supplementary Fig. 14a) to generate 3-bp insertions at EMX1, HEK1 and LSP1 genomic sites (Fig. 2e). Deep sequencing showed that PE3 induced $\sim 30 \%-70 \%$ intended insertion frequencies (Fig. 2f) at the on-target sites. WGS of the PE3-edited single cell colonies (Supplementary Fig. 15a-15c) displayed that PE3 induced similar or fewer indels (Fig. $2 \mathrm{~g}$ and Supplementary Fig. 16a) and base substitutions (Fig. 2h and Supplementary Fig. 16b), comparing to Cas9 or EGFP 
control. Meanwhile, the designed 3-bp insertions were not found at OT sites on a genome-wide scale (Supplementary Fig. 17a-17c).

Furthermore, we transfected different amounts of plasmids to express PE, pegRNA and nicking gRNA or transfected same amounts of plasmids but for different exposure time periods. In all tested conditions, PE3 manifested no more genome-wide OT mutations than the EGFP control (Supplementary Fig. 18a-18c). We also examined whether PE3 copied pegRNA scaffold into genome and found that no pegRNA sequence was inserted into the genomic DNA (Supplementary Fig. 19a and 19b). As the effector moiety of PE3 is an RTase, we therefore determined whether PE3 affected telomere integrity in PE3-treated single cell colonies and discovered that the length and sequence of telomeric region were barely affected by PE3

(Supplementary Fig. 20a-20d). After examing the gRNA-independent OT effects genome-wide, we also determined the gRNA-dependent OT mutations by PE3 in WT 293FT cells. No obvious base substitution (Supplementary Fig. 21a and 21b) or indel (Supplementary Fig. 22a and 22b) was induced by PE3 at gRNA-dependent OT sites $^{18,19}$. Nevertheless, it is worthwhile noting that although PE3 induced relatively lower levels of unintended indels at on-target sites than Cas9, the average unintended indel frequencies induced by PE3 at on-target sites were $\sim 10 \%$ (Supplementary Fig. $3 \mathrm{~b}, 10 \mathrm{~b}$ and $14 \mathrm{~b})$. It is likely due to the requirement of both pegRNA and nicking gRNA in the PE3 system, which may cleave opposite DNA strands near on-target site 
to generate a DSB, and the repair of resulted DSB could then lead to small indels or possibly, large genomic deletions ${ }^{20}$.

In addition to gRNA-independent OT effects on genomic DNA, gRNA-independent OT effects of genome editors on transcriptomic RNA have been recently reported as well ${ }^{6,8}$. Thus, we performed whole-transcriptome sequencing and used the RADAR pipeline $^{21}$ to detect whether PE3 induced gRNA-independent OT mutations in transcriptomic RNA in WT 293FT cells, with EGFP and Cas9 as negative controls and hA3A-BE3 as a positive control. Whole-transcriptome sequencing results demonstrated that comparing to EGFP or Cas9, PE3 induced similar mutations at the transcriptomic RNA level (Fig. 3a, 3b, 3c and 3e). In contrast, hA3A-BE3 induced much more mutations in transcriptomic RNA than EGFP, Cas9 and PE3 (Fig. 3a, 3b and 3d), and most of mutations were C-to-U substitutions (Fig. 3a), which were catalyzed by the hA3A deaminase moiety of hA3A-BE3 in a gRNA-independent manner. Furthermore, we also examined whether PE treatment affects gene expression and found that expression patterns in PE-treated and EGFP-treated cells were similar, suggesting that PE did not affect gene expression (Supplementary Fig. $23 a$ and $23 b)$. 
Collectively, we evaluated gRNA-independent OT mutations of PE3 by performing whole-genome and whole-transcriptome sequencing and showed that PE3 induced no observable OT mutation in a gRNA-independent manner, when generating three types of targeted edits, i.e., base substitution, small deletion and insertion. Together with previous studies showing the versatility and high efficiency of PE's on-target editing ${ }^{1,}$ ${ }^{2}$, our results highlighted the high editing specificity of PE system. 


\section{METHODS}

\section{Plasmid construction}

Oligonucleotides hRNF2_FOR/hRNF2_REV were annealed and ligated into BsaI linearized pGL3-U6-gRNA-PGK-EGFP to generate the vector psgRNF2 for the expression of sgRNF2. Oligonucleotides +41_nicking-hRNF2_FOR/+41_nickinghRNF2 _REV were annealed and ligated into BsaI linearized pGL3-U6-gRNA-PGKpuromysin to generate the vector pnsgRNF2 for the expression of +41 nicking sgRNF2. Other gRNA and nicking gRNA expression vectors were constructed by a similar strategy, respectively.

The primer sets (pegRNF2_F/pegRNF2_R) were used to amplify the fragment scaffold-pegRNF2 with the template pU6-pegRNA-GG-Vector ${ }^{1}$.Then, the amplified fragment scaffold-pegRNF2 was cloned into BsaI and EcoRI linearized pU6pegRNA-GG-Vector to generate the vector pU6-pegRNF2. Other pegRNA expression vectors were constructed by a similar strategy, respectively.

The sequences of the oligos used for plasmid construction are listed in Supplementary Table 1.

Establishment of 293FT ${ }^{A 3-/-}$ cells 
To establish the APOBEC3-knockout (293FT $\left.{ }^{A 3-/-}\right)$ cell line, 293FT cells were seeded into a 60 -mm plate at a density of $4 \times 10^{5}$ per well and cultured for 24 hours. The cells were then transfected with a plasmid expressing Cas9 nuclease and a plasmid expressing two gRNAs targeting the upstream genomic region of $A P O B E C 3 A$ and the downstream genomic region of $A P O B E C 3 H$ (sghA3A-up and sghA3H-down,

Supplementary Fig. 1a), with a puromycin-resistant selection gene. After 48 hours, 10 $\mu \mathrm{g} / \mathrm{ml}$ puromycin was added into the media to enrich the transfected cells and after two weeks of enrichment, single cell colonies were sorted into 96-well plates without puromycin for colony expansion. The non-homologous end joining of two DNA ends generated by Cas 9 and sghA3A-up/sghA3H-down can remove the whole gene cluster of $A P O B E C 3$ from the genome of $293 \mathrm{FT}$ cells and the single cell colony containing successful knockout of $A P O B E C 3$ cluster was validated by genomic DNA PCR with the primers flanking the Cas9 cleavage sites (Supplementary Fig. 1b and 1c). RT$\mathrm{qPCR}^{22}$ further confirmed that the expression of all $A P O B E C 3$ genes was not detected in 293FT ${ }^{A 3-/-}$ cells (Supplementary Fig. 1d).

\section{Cell culture and transfection for on-target and gRNA-dependent off-target deep-}

\section{sequencing}

293FT from ATCC or 293FT ${ }^{A 3-/-}$ cells were maintained in DMEM (10566,

Gibco/Thermo Fisher Scientific) + 10\% FBS (16000-044, Gibco/Thermo Fisher

Scientific) and regularly tested to exclude mycoplasma contamination. 
Before transfection, cells were seeded in a 24 -well plate at a density of $1 \times 10^{5}$ per well. For base editing with hA3A-BE3 and gene editing with Cas9, the cells were transfected with $250 \mu 1$ serum-free Opti-MEM that contained $2.52 \mu 1$ LIPOFECTAMINE LTX (Life, Invitrogen), $0.84 \mu$ LIPOFECTAMINE plus (Life, Invitrogen), $0.5 \mu \mathrm{g}$ pCMV-hA3A-BE3 (or pCMV-spCas9) expression vector and 0.34 $\mu \mathrm{g}$ gRNA expression vector. For prime editing with PE3, the cells were transfected with $250 \mu$ l serum-free Opti-MEM that contained $3.9 \mu$ LIPOFECTAMINE LTX, 1.3 $\mu 1$ LIPOFECTAMINE plus, $0.9 \mu \mathrm{g}$ PE3 expression vector, $0.3 \mu \mathrm{g}$ pegRNA expression vector and $0.1 \mu \mathrm{g}$ nicking gRNA expression vector. For EGFP expression, the cells were transfected with $250 \mu 1$ serum-free Opti-MEM that contained $1.5 \mu 1$ LIPOFECTAMINE LTX (Life, Invitrogen), $0.5 \mu$ LIPOFECTAMINE plus (Life, Invitrogen), $0.5 \mu \mathrm{g}$ pCMV-EGFP expression vector. 72 hours after transfection, transfected cells in the first $10 \%$ of the fluorescence intensity were sorted by BD FACSAria III, from which the genomic DNA was extracted with QuickExtract ${ }^{\mathrm{TM}}$ DNA Extraction Solution (QE09050, Epicentre) for subsequent sequencing analysis.

\section{Isolation and expansion of edited single cell colonies for whole-genome}

\section{sequencing}

$293 \mathrm{FT}^{A 3-/-}$ cells expanded from a single cell colony with successful $A 3$ knockout were maintained in DMEM (10566, Gibco/Thermo Fisher Scientific) + 10\% FBS (16000- 
044, Gibco/Thermo Fisher Scientific) and regularly tested to exclude mycoplasma contamination. The single cell colony-derived $293 \mathrm{FT}^{A 3-/}$ cells were transfected with genome editors (e.g., PE3, hA3A-BE3 and Cas9) or EGFP-expressing plasmids and 72 hours after transfection, single cells were sorted into 96-well plates by BD FACSAria III. After 18-day colony expansion, the genomic DNA derived from transfected single cell colonies was extracted with QuickExtract ${ }^{\mathrm{TM}}$ DNA Extraction Solution (QE09050, Epicentre) for Sanger sequencing and the genomic DNA with biallelic editing was further subjected to whole-genome sequencing. On average, one bi-allelic edited colony can be obtained from $\sim 6-8$ colonies when the editing efficiency of bulk setting is $\sim 30-50 \%$.

\section{DNA library preparation and sequencing}

Target genomic sequences were PCR amplified by high-fidelity DNA polymerase

PrimeSTAR HS (Clonetech) with primer sets flanking examined gRNA target sites.

The gRNA target sequences and PCR primer sequences are listed in Supplementary

Table 2. Indexed DNA libraries were prepared by using the NEBNext Ultra II FS

DNA Library Prep Kit for Illumina. After quantitated with Qubit High-Sensitivity

DNA kit (Invitrogen), PCR products with different tags were pooled together for deep sequencing by using the Illumina Hiseq X Ten $(2 \times 150)$ at CAS-MPG Partner Institute for Computational Biology Omics Core, Shanghai, China. Raw read qualities were evaluated by FastQC (v0.11.8, 
http://www.bioinformatics.babraham.ac.uk/projects/fastqc/, parameters: default). For paired-end sequencing, only R1 reads were used. Adaptor sequences and read sequences with Phred quality score lower than 30 were trimmed. Trimmed reads were then mapped with the BWA-MEM algorithm (BWA v0.7.17) to target sequences. After piled up with Samtools (v1.9), base substitutions and indel frequencies at ontarget sites were calculated according to previously published literature ${ }^{13,23}$.

\section{Base substitution frequency calculation}

Base substitution of every position at the target sites of examined gRNAs and pegRNAs was piled up with at least 1000 independent reads. Base substitution frequencies were calculated as: (count of reads with substitution at the target base)/(count of reads covering the target base). Counts of reads for each base at examined target sites and gRNA-dependent OT sites are listed in Supplementary Table 3 and 5, respectively.

\section{Intended indel frequency calculation}

Intended indel frequencies were calculated as: (count of reads with only intended indel at the target site)/(count of total reads covering the target site). These counts are listed in Supplementary Table 4.

\section{Unintended indel frequency calculation}


Unintended indel frequencies for base substitution were estimated among reads aligned in the region spanning from upstream 8 nucleotides to the target site (or gRNA-dependent OT site) to downstream 19 nucleotides to PAM site (50 bp). Unintended indel frequencies for base substitution were calculated as: (count of reads containing at least one unintended inserted and/or deleted nucleotide)/(count of total reads aligned in the estimated region). While unintended indel frequencies for targeted insertion/deletion were estimated among reads aligned at the target site. Unintended indel frequencies for targeted insertion/deletion were calculated as: (count of reads containing unintended indels)/(count of total reads covering the target site). The counts for on-target and gRNA-dependent OT sites are listed in Supplementary Tables 4 and 5, respectively.

\section{Whole-genome sequencing and data analysis}

Genomic DNA was extracted from transfected $293 \mathrm{FT}^{A 3-/}$ single cell colonies by using cell DNA isolation kit FastPure ${ }^{\circledR}$ (DC102-01, Vazyme). Indexed DNA libraries were prepared by using NEBNext Ultra II FS DNA Library Prep Kit for Illumina. A total of $12 \mathrm{~Tb}$ WGS data were obtained by using Illumina Hiseq X Ten $(2 \times 150)$ at CASMPG Partner Institute for Computational Biology Omics Core, Shanghai, China. The average coverage of sequencing data generated for each transfected $293 \mathrm{FT}^{A 3-/-}$ single cell colony sample was $14 \times$, with a minimum depth at $12 \times$. These WGS datasets were individually analyzed with a $\underline{B} a s e / P r i m e ~ \underline{e d i t o r}$ induced $\underline{\mathrm{DNA}} \underline{\mathrm{o} f f-t a r g e t}$ site 
identification unified toolkit (BEIDOU, https://github.com/YangLab/BEIDOU) to call high-confident base substitution or indel events that could be identified by all three different callers, GATK ${ }^{24}$, Lofreq ${ }^{25}$ and Strelka2 ${ }^{26}$.

Briefly, to reduce the impact of varying sequence depth among samples, 120M reads were randomly sampled by Seqtk (v1.3, https://github.com/lh3/seqtk, parameters: sample -s100 120000000) from raw data for further analyses. After quality control by FastQC (parameters: default), WGS DNA-seq reads were trimmed by Trimmomatic (v0.38, parameters: ILLUMINACLIP:TruSeq3-PE-2.fa: 2:30:10 LEADING:3 TRAILING:3 SLIDINGWINDOW:4:15 MINLEN:36) ${ }^{27}$ to remove low quality read sequence. BWA-MEM algorithm (v0.7.17, parameters: default) was used to map clean reads to the human reference genome (hg38). Samtools (v1.9, parameters: -bh F 4 -q 30) was used to select reads with mapping quality score $\geqslant 30$ and convert SAM files to sorted BAM files. After marking duplicate reads by Picard (v2.21.2, parameters: REMOVE_DUPLICATES=false) in the BAM file, GATK (v4.1.3.0) was employed to correct systematic bias by a two-stage process (BaseRecalibrator and ApplyBQSR, parameters: default).

Single nucleotide variations of OT mutations were individually computed by the BEIDOU toolkit with three algorithms GATK, Lofreq (v2.1.3.1, parameters: default) and Strelka2 (v2.9.10, parameters: default) with workflows for the germline variant 
calling. Genome-wide indels were also detected by the BEIDOU toolkit with GATK, Strelka2 (parameters: default) and Scalpel (v0.5.4, parameters: --single --window $600)^{28}$. For GATK, genome-wide de novo variants were determined by three GATK commands, HaplotypeCaller (parameters: default), VariantRecalibrator (parameters: “--resource:hapmap,known=false, training=true,truth=true,prior=15.0 hapmap_3.3.hg38.vcf.gz -resource:omni,known=false,training=true, truth=false,prior $=12.0$ 1000G_omni2.5.hg38.vcf.gz -resource: $1000 \mathrm{G}, \mathrm{known}=$ false,training $=$ true,truth=false, prior $=10.0$ 1000G_phase1.snps.high_confidence.hg38.vcf.gz -resource:dbsnp,known=true, training=false, truth $=$ false, prior $=2.0$ dbsnp_146.hg38.vcf.gz -an QD -an MQ -an MQRankSum -an ReadPosRankSum -an FS -an SOR -an DP --max-gaussians 4” for SNVs; “resource:mills,known=true, training=true,truth=true, prior $=12.0$ Mills_and_1000G_gold_standard.indels.hg38.vcf.gz -an QD -an MQRankSum -an ReadPosRankSum -an FS -an SOR -an DP --max-gaussians 4 -mode INDEL” for indels) and ApplyVQSR (parameters: “-mode SNP -ts-filter-level 95” for SNVs; “mode INDEL -ts-filter-level 95" for indels). VCF files used for VariantRecalibrator were downloaded from https://ftp.ncbi.nih.gov/snp/ and https://console.cloud.google.com/storage/browser/genomics-publicdata/resources/broad/hg38/v0. Of note, overlaps of three algorithms of SNVs/indels 
were considered as reliable variants by the BEIDOU toolkit. To further obtain de novo SNVs/indels, we filtered out the background variants, including: (1)

SNVs/indels in non-transfected cells of this study and dbSNP (v151,

http://www.ncbi.nlm.nih.gov/SNP/) database; (2) SNVs/indels with allele frequencies less than $10 \%$ or depth less than 10 reads; (3) SNVs/indels overlapped with the UCSC repeat regions. Analyses were only focused on SNVs/indels from canonical (chr 1-22, $\mathrm{X}, \mathrm{Y}$ and $\mathrm{M}$ ) chromosomes. Genome-wide base substitutions and indels are listed in Supplementary Table 6 .

\section{Predication of gRNA-dependent OT site}

Potential gRNA-dependent OT sites were predicted by Cas-OFFinder ${ }^{19}$, allowing up to 5 mismatches. OT sites identified by WGS shown in Supplementary Fig. 7 were randomly selected from genome-wide base substitutions.

\section{Telomere length calculation and variant calling from WGS data}

Telseq ${ }^{29}$ (parameters: -k 2) was used to calculate the telomere lengths. BAM files containing mapped WGS DNA-seq reads processing from the BEIDOU toolkit were as the input of Telseq. Telomeric repeat variants, the sequence fragments within telomeric reads that differ from the canonical telomeric repeat pattern (TTAGGG in human), were identified by Computel $^{30}$ (v1.2, parameters: default) with trimmed 
WGS fastq files as input. Telomere length and the numbers of telomeric repeat variants are listed in Supplementary Table 7.

\section{RNA extraction and whole-transcriptome sequencing and data analysis}

After 48 hours of transfection with genome editors (e.g., PE3, hA3A-BE3 and Cas9) or EGFP-expressing plasmids, transfected cells in the first $10 \%$ of the fluorescence intensity were sorted by BD FACSAria III. Total RNAs of sorted cells were extracted by using the RNeasy Mini Kit (QIAGEN \#74104) for whole-transcriptome sequencing. RNA-seq libraries were prepared using Illumina TruSeq Stranded Total RNA LT Sample Prep Kit. Size-selected libraries were subjected to deep sequencing with Illumina Hiseq X Ten $(2 \times 150)$. Raw read qualities were evaluated by FastQC (http://www.bioinformatics.babraham.ac.uk/projects/fastqc/). RNA editing sites were called by the published RADAR pipeline ${ }^{21}$. Gene expression was determined by FPKM (Fragments Per Kilobase of transcript per Million mapped reads) with featureCounts (v1.6.3, --fraction -O -t exon -g gene_id). Transcriptome-wide mutations are listed in Supplementary Table 8.

\section{Data Availability}

Deep sequencing data and WGS data can be accessed in the NCBI Gene Expression Omnibus (accession no. xxxx) and the National Omics Data Encyclopedia (accession no: OEPxxxxxx. Of note, the accession code will be available before publication). 


\section{Code Availability}

The BEIDOU tookit that calls high-confident base substitution or indel events from WGS data is available at https://github.com/YangLab/BEIDOU. The custom Python, Perl and Shell scripts for base substitution and indel frequencies calculation will be available upon request.

\section{Statistical analysis}

All statistical analyses were performed with R package 3.6.2 (http://www.R-

project.org/). $P$ values were calculated from one-tailed Student's $t$ test in this study. 


\section{ACKNOWLEDGMENTS}

This work was supported by grants 2019YFA0802804 (L.Y.), 2018YFA0801401

(J.C.) and 2018YFC1004602 (J.C.) from MoST, 31925011 (L.Y.), 91940306 (L.Y.),

31822016 (J.C.) and 81872305 (J.C.) from NSFC. We thank Molecular and Cell

Biology Core Facility, School of Life Science and Technology, ShanghaiTech

University for providing experimental service.

\section{AUTHOR CONTRIBUTIONS}

J.C., L.Y. and X.H. conceived, designed and supervised the project. R.G. and X.L.

performed most experiments with the help of G.L., L.W. and J.Wu on cell culture and plasmid construction. J.Wei prepared libraries for DNA sequencing and Z-C.F. and Y.W. performed bioinformatics analyses with the help of W.X., supervised by L.Y.

J.C. and L.Y. wrote the paper with inputs from the authors. J.C. managed the project.

\section{DECLARATION OF INTERESTS}

The authors declare no competing interests. 


\section{REFERENCES}

1. Anzalone, A.V. et al. Search-and-replace genome editing without doublestrand breaks or donor DNA. Nature 576, 149-157 (2019).

2. $\quad$ Lin, Q. et al. Prime genome editing in rice and wheat. Nat Biotechnol 38, 582585 (2020).

3. Yang, L., Yang, B. \& Chen, J. One Prime for All Editing. Cell 179, 1448-1450 (2019).

4. Anzalone, A.V., Koblan, L.W. \& Liu, D.R. Genome editing with CRISPR-Cas nucleases, base editors, transposases and prime editors. Nat Biotechnol 38, 824-844 (2020).

5. Kim, D.Y., Moon, S.B., Ko, J.H., Kim, Y.S. \& Kim, D. Unbiased investigation of specificities of prime editing systems in human cells. Nucleic Acids Res 48, 10576-10589 (2020).

6. Grunewald, J. et al. Transcriptome-wide off-target RNA editing induced by CRISPR-guided DNA base editors. Nature 569, 433-437 (2019).

7. Jin, S. et al. Cytosine, but not adenine, base editors induce genome-wide offtarget mutations in rice. Science 364, 292-295 (2019).

8. Zhou, C. et al. Off-target RNA mutation induced by DNA base editing and its elimination by mutagenesis. Nature 571, 275-278 (2019).

9. Zuo, E. et al. Cytosine base editor generates substantial off-target singlenucleotide variants in mouse embryos. Science 364, 289-292 (2019). 
10. Burns, M.B. et al. APOBEC3B is an enzymatic source of mutation in breast cancer. Nature 494, 366-370 (2013).

11. Burns, M.B., Temiz, N.A. \& Harris, R.S. Evidence for APOBEC3B mutagenesis in multiple human cancers. Nat Genet 45, 977-983 (2013).

12. Lei, L. et al. APOBEC3 induces mutations during repair of CRISPR-Cas9generated DNA breaks. Nat Struct Mol Biol 25, 45-52 (2018).

13. Wang, X. et al. Efficient base editing in methylated regions with a human APOBEC3A-Cas9 fusion. Nat Biotechnol 36, 946-949 (2018).

14. Jin, S. et al. Rationally Designed APOBEC3B Cytosine Base Editors with Improved Specificity. Mol Cell 79, 728-740 e726 (2020).

15. Zuo, E. et al. A rationally engineered cytosine base editor retains high ontarget activity while reducing both DNA and RNA off-target effects. Nat Methods 17, 600-604 (2020).

16. Chen, J., Yang, B. \& Yang, L. To BE or not to BE, that is the question. Nat Biotechnol 37, 520-522 (2019).

17. Yang, L. \& Chen, J. A Tale of Two Moieties: Rapidly Evolving CRISPR/CasBased Genome Editing. Trends Biochem Sci 45, 874-888 (2020).

18. Tsai, S.Q. et al. GUIDE-seq enables genome-wide profiling of off-target cleavage by CRISPR-Cas nucleases. Nat Biotechnol 33, 187-197 (2015). 
19. Bae, S., Park, J. \& Kim, J.S. Cas-OFFinder: a fast and versatile algorithm that searches for potential off-target sites of Cas9 RNA-guided endonucleases.

Bioinformatics 30, 1473-1475 (2014).

20. Kosicki, M., Tomberg, K. \& Bradley, A. Repair of double-strand breaks induced by CRISPR-Cas9 leads to large deletions and complex rearrangements. Nat Biotechnol 36, 765-771 (2018).

21. Wang, X. et al. Cas12a Base Editors Induce Efficient and Specific Editing with Low DNA Damage Response. Cell Rep 31, 107723 (2020).

22. Chen, J., Miller, B.F. \& Furano, A.V. Repair of naturally occurring mismatches can induce mutations in flanking DNA. Elife 3, e02001 (2014).

23. Li, X. et al. Base editing with a Cpf1-cytidine deaminase fusion. Nat Biotechnol 36, 324-327 (2018).

24. McKenna, A. et al. The Genome Analysis Toolkit: a MapReduce framework for analyzing next-generation DNA sequencing data. Genome Res 20, 1297-1303 (2010).

25. Wilm, A. et al. LoFreq: a sequence-quality aware, ultra-sensitive variant caller for uncovering cell-population heterogeneity from high-throughput sequencing datasets. Nucleic Acids Res 40, 11189-11201 (2012).

26. Kim, S. et al. Strelka2: fast and accurate calling of germline and somatic variants. Nat Methods 15, 591-594 (2018). 
27. Bolger, A.M., Lohse, M. \& Usadel, B. Trimmomatic: a flexible trimmer for Illumina sequence data. Bioinformatics 30, 2114-2120 (2014).

28. Narzisi, G. et al. Accurate de novo and transmitted indel detection in exomecapture data using microassembly. Nat Methods 11, 1033-1036 (2014).

29. Ding, Z. et al. Estimating telomere length from whole genome sequence data. Nucleic Acids Res 42, e 75 (2014).

30. Nersisyan, L. \& Arakelyan, A. Computel: computation of mean telomere length from whole-genome next-generation sequencing data. PLoS One 10, e0125201 (2015). 


\section{FIGURES AND FIGURE LEGENDS}

a

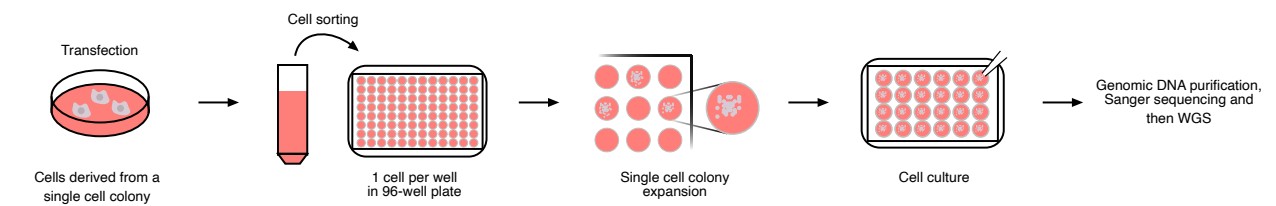

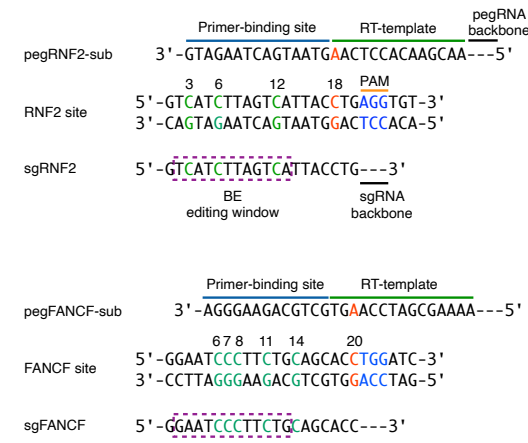

pegSEC61B-sub $\quad 3^{\prime}-$ Primer-binding site $^{- \text {TAACGAAAGTTTCAAGACCATACTTGTG---5' }} \frac{\text { 'template }}{}$

$\stackrel{2}{2} \stackrel{9}{9} 13 \quad 24$

-

sgSEC61B 5' -A'CTGATTGGCTTTCAAAGTTC---3'
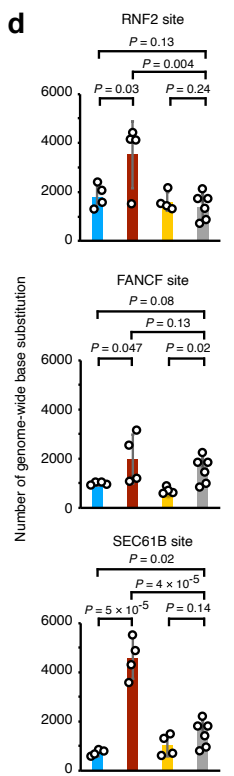

e

2000 c

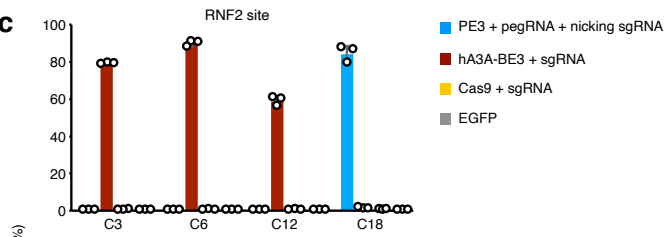

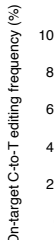
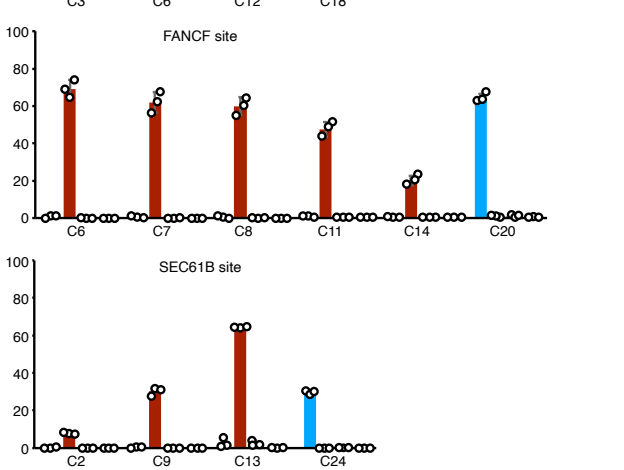

RNF2 site

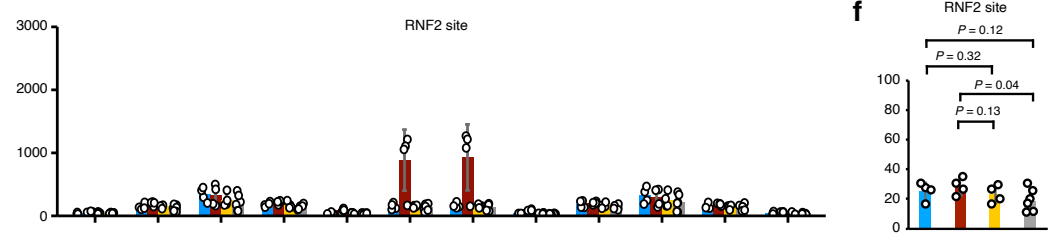

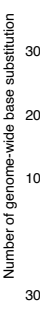
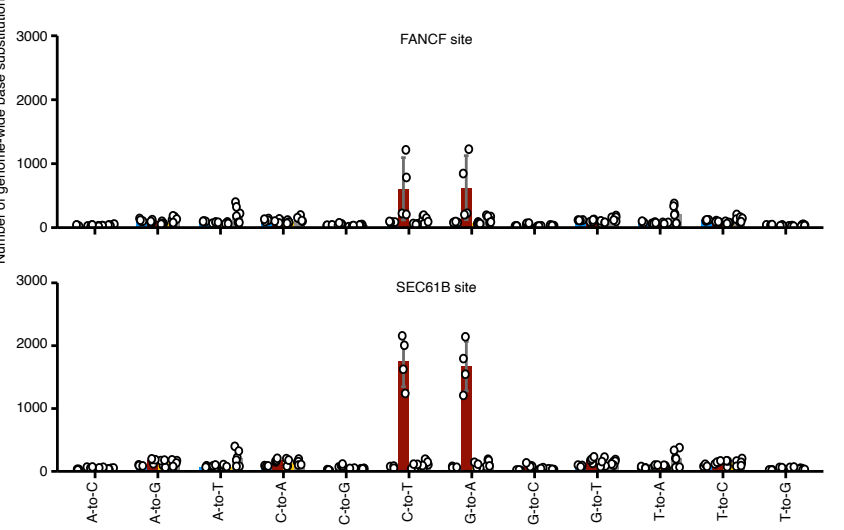
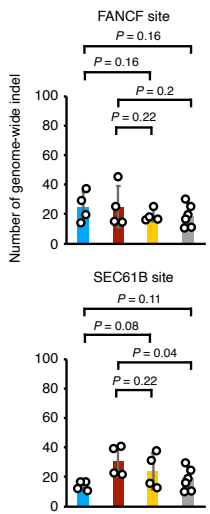

Fig. 1. PE3 induced no observable genome-wide OT mutation when generating

\section{base substitutions.}

(a) Schematic diagrams illustrate the procedure to determine genome-wide OT

mutations in edited single cell colonies. Briefly, 293FT ${ }^{A 3-/-}$ cells were transfected with 
genome editors and after transfection, single cells were sorted into 96-well plates by cell sorter. After colony expansion, the genomes derived from single cell colonies were extracted for Sanger sequencing and then the ones with bi-allelic editing were further subjected to WGS. (b) Schematic diagrams illustrate $R N F 2, F A N C F$ and SEC61B target sites, the spacer sequences of gRNAs and the primer-binding sites and reverse transcription (RT) templates of pegRNAs. The editing sites of PE3 and hA3ABE3 are in red and green respectively. (c) C-to-T editing frequencies (count of reads with C-to-T editing at the target base/count of reads covering the target base) induced by the indicated genome editors at target sites. Means \pm s.d. were from three independent experiments. (d) Numbers of genome-wide base substitutions induced by indicated genome editors in single cell colonies with bi-allelic edits at on-target sites shown in Supplementary Fig. 4. (e) Numbers of each type of base substitution induced by indicated genome editors shown in (d). (f) Numbers of genome-wide indels induced by indicated genome editors in single cell colonies shown in Supplementary Fig. 4. (d) - (f) Means \pm s.d. were from four or six (EGFP) independent colonies. $P$ value, one-tailed Student's $t$ test. 
a

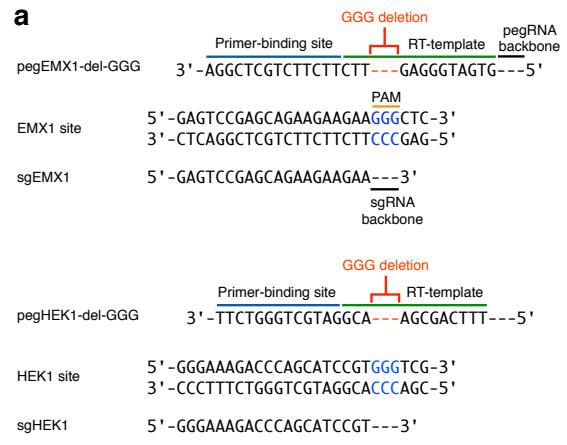

pegLSP1-del-TGC $\quad 3^{\prime}$-ACTCTTCCGTTGTG---GCCGACCCCGAC---5'

LSP1 site 5' -CTGTGAGAAGGCACACTGCCGGCTG-3'

sgLSP1 5 '-CTGTGAGAAGGCAACACTGC---3'

e

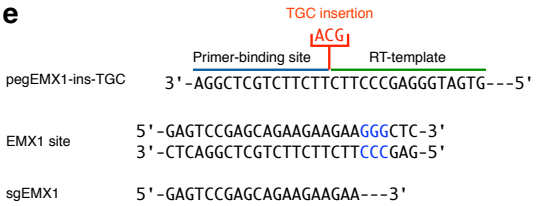

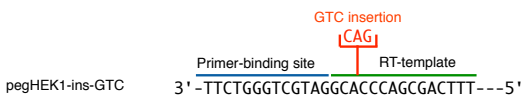

HEK1 site 5' -GGGAAAGACCCAGCATCCGTGGGTGG-3'

3' -'CCCTTTCTGGGTCGTAGGCACCCAGC-5'

sgHEK1 5 ' -GGGAAAGACCCAGCATCCGT---3'

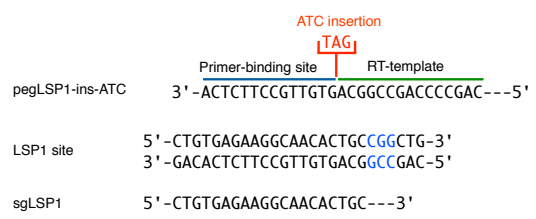

b
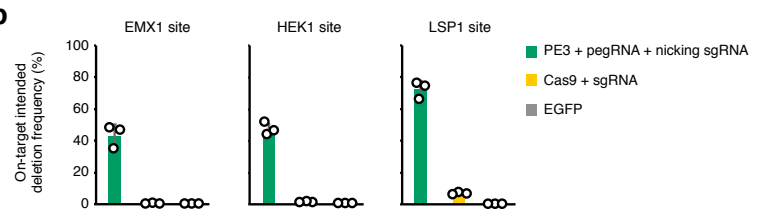

c
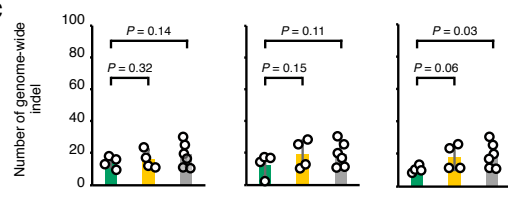

d
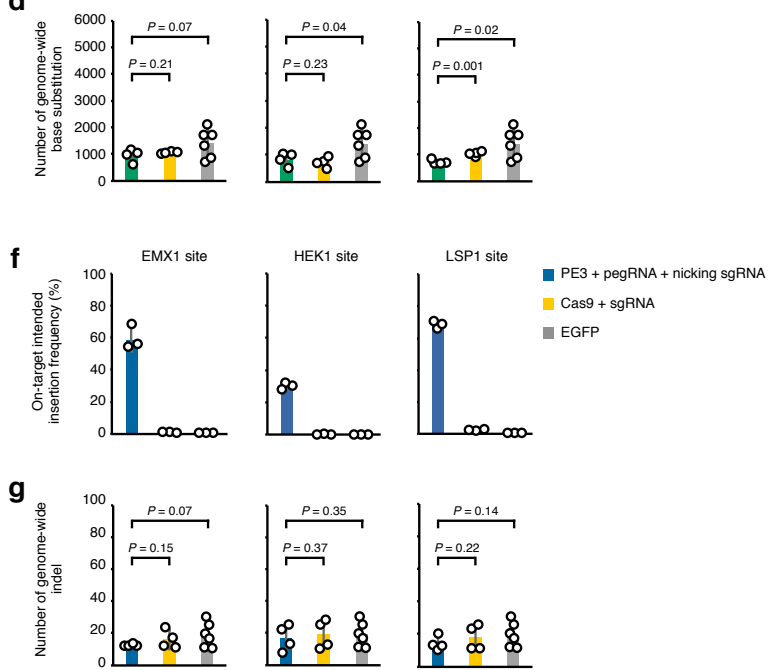

h

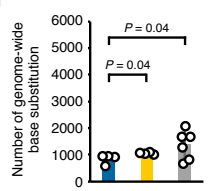

Fig. 2. PE3 induced no observable genome-wide OT mutation when generating

\section{small deletions and insertions.}

(a) Schematic diagrams illustrate EMX1, HEK1 and LSP1 target sites, the spacer sequences of gRNAs and the primer-binding sites and RT templates of pegRNAs for targeted deletions. The designed deletions in pegRNA are in red. (b) Targeted deletion frequencies induced by the indicated genome editors at on-target sites. (c), (d) Numbers of genome-wide indels (c) and base substitutions (d) induced by indicated genome editors in single cell colonies shown in Supplementary Fig. 11. (e) 
Schematic diagrams illustrate $E M X 1, H E K 1$ and $L S P 1$ target sites, the spacer sequences of gRNAs and the primer-binding sites and RT templates of pegRNAs for targeted insertions. The designed insertions in pegRNA are in red. (f) Targeted insertion frequencies induced by the indicated genome editors at on-target sites. (g), (h) Numbers of genome-wide indels (g) and base substitutions (h) induced by indicated genome editors in single cell colonies shown in Supplementary Fig. 15. The data for Cas9 and EGFP in (f)-(h) are same as the ones in (b)-(d). (b) and (f) Means \pm s.d. were from three independent experiments. (c), (d), (g) and (h) Means \pm s.d. were from four or six (EGFP) independent colonies. $P$ value, one-tailed Student's $t$ test. 
a
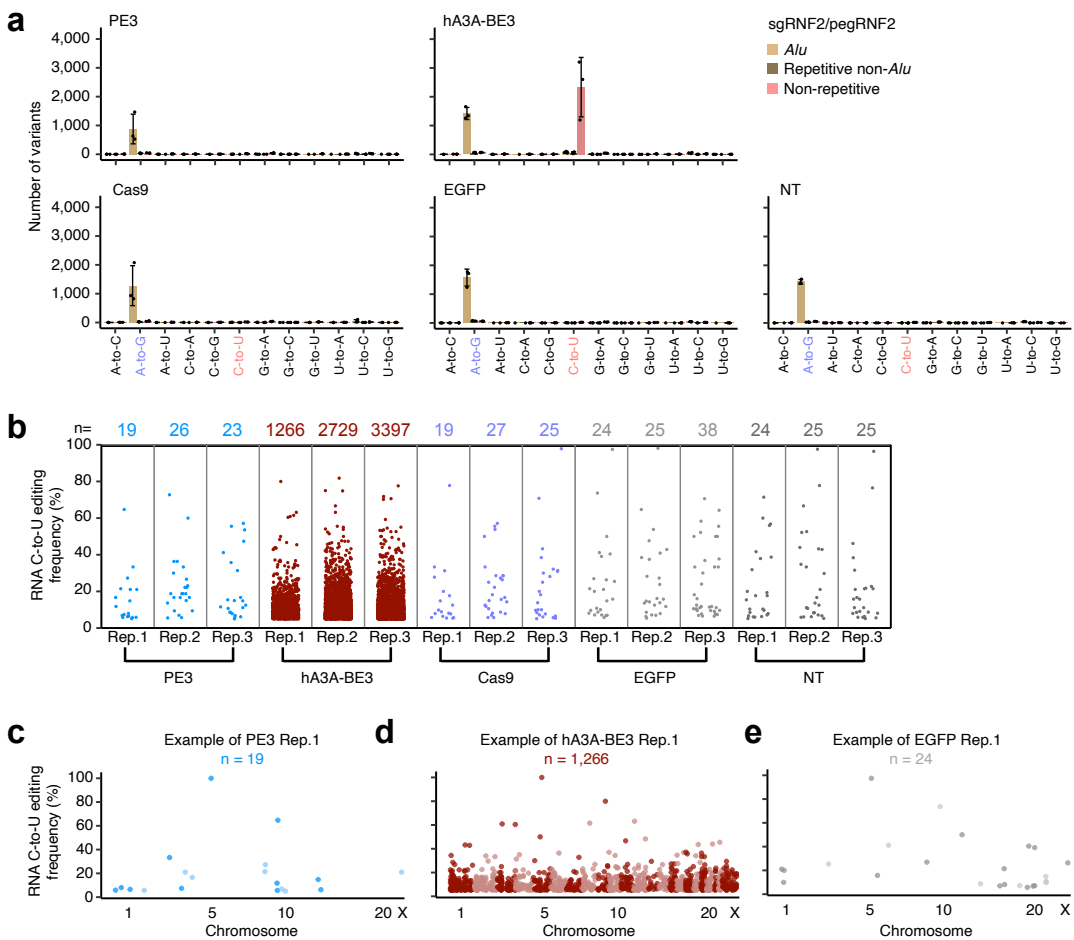

Fig. 3. PE3 induced no observable transcriptome-wide OT mutation.

(a) Histogram to show numbers of all 12 types of RNA editing in different regions from cells treated with PE3, hA3A-BE3, Cas9, EGFP or left non-transfected (NT).

RNA editing was analyzed and visualized by the RADAR pipeline. Means \pm s.d. were from three independent experiments. (b) Manhattan plot of RNA off-target editing (C-to-U) frequency shown in (a). (c, d, e) The RNA off-target editing frequencies and sites induced by PE3 replicate $1(\mathbf{c})$, hA3A-BE3 replicate 1 (d) or EGFP replicate 1 (e). 


\section{SUPPLEMENTARY FIGURES AND LEGENDS}

a

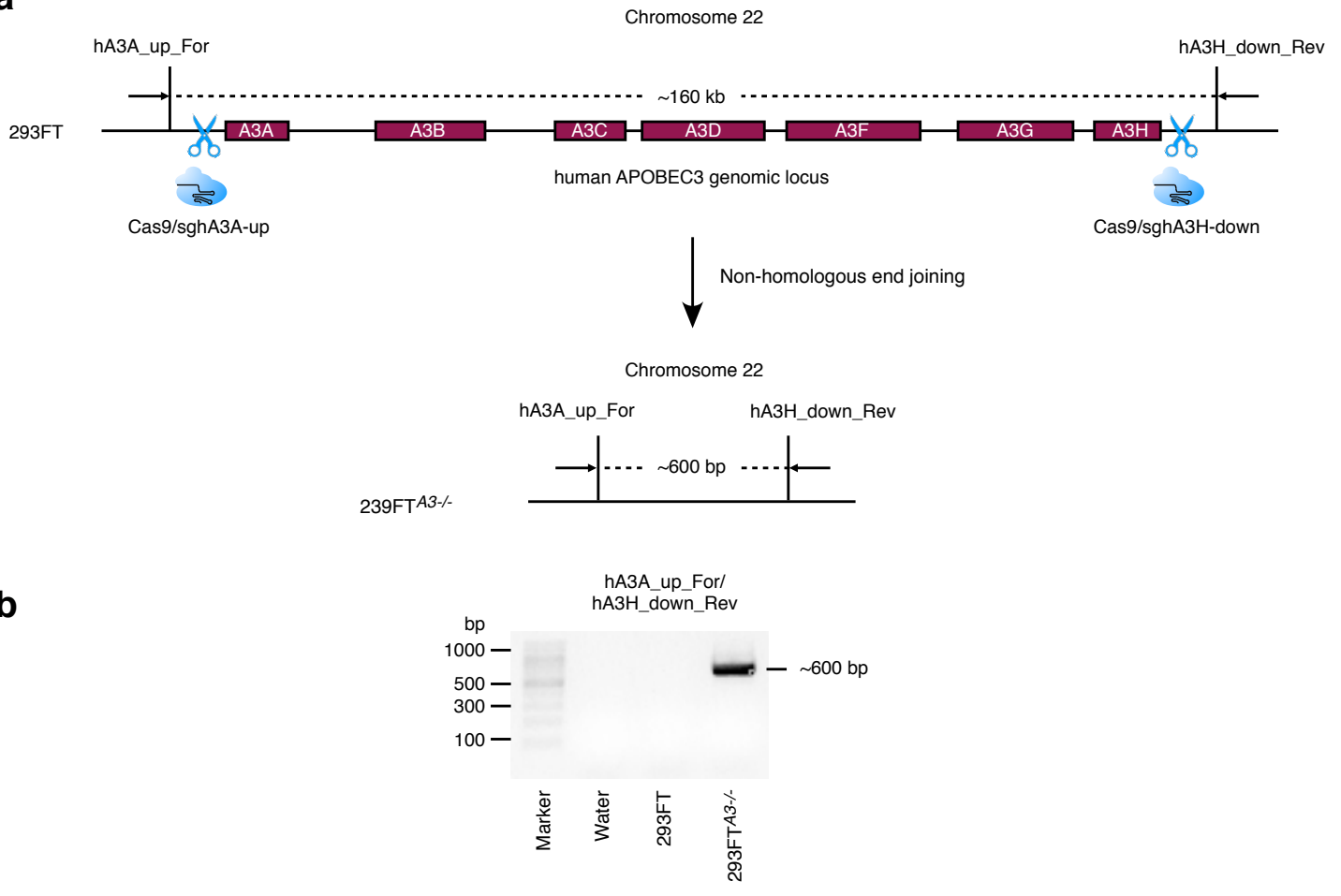

C

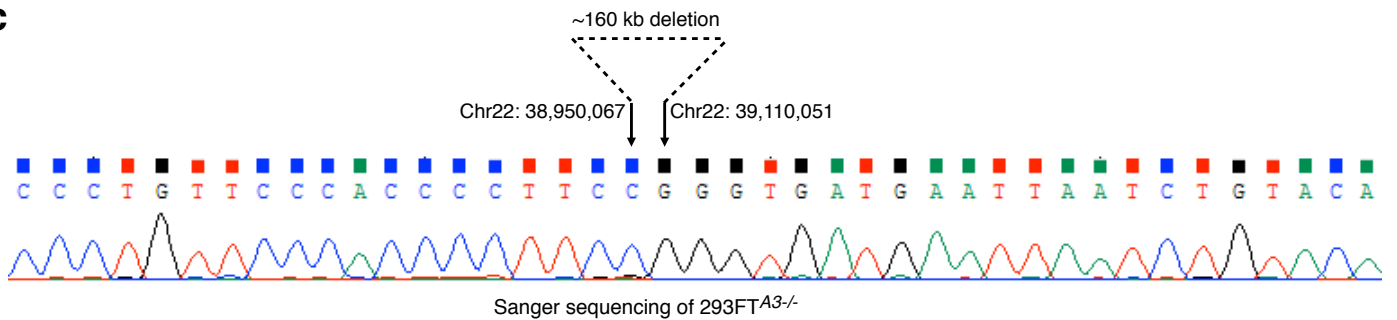

d
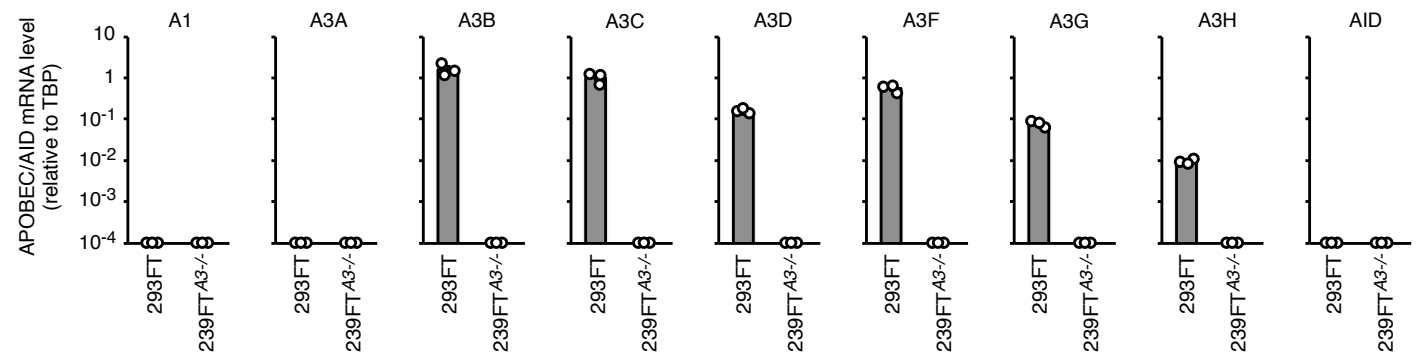

\section{Supplementary Fig. 1. Generation of $A P O B E C 3$-knockout 293FT cell line.}

(a) Schematic diagrams illustrate the strategy to delete $A P O B E C 3$ gene cluster from the genome of 293FT cells. (b) Validation of APOBEC3-knockout by RT-PCR. The non-homologous end joining of two Cas9 cleavage site results in a PCR-detectable 
band ( 600 bp). (c) Validation of $A P O B E C 3$-knockout by Sanger sequencing of the PCR amplified band shown in (b). (d) Relative APOBEC/AID mRNA expression levels in $A P O B E C 3$-knockout cells and wildtype cells. Means \pm s.d. were from three independent colonies. 
a

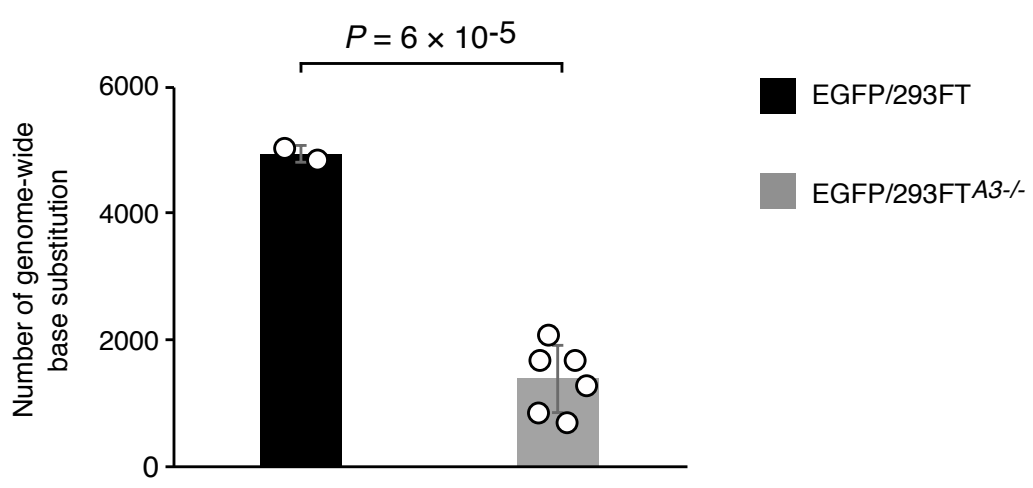

b

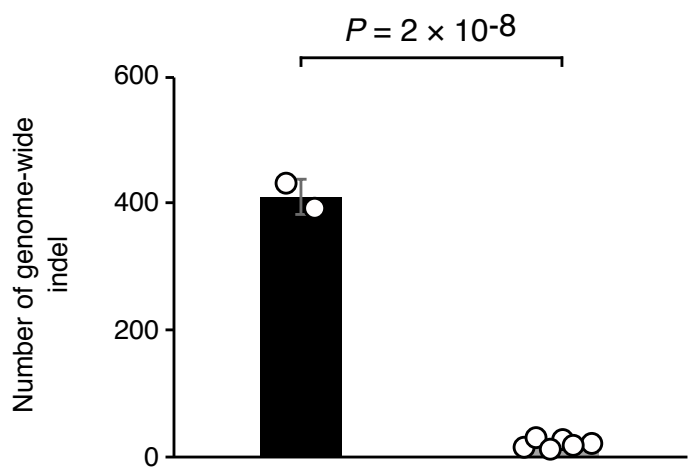

Supplementary Fig. 2. Knockout of $A P O B E C 3$ gene cluster reduced genomic mutation background.

(a) Comparison of numbers of genome-wide base substitutions in WT 293FT and 293FT ${ }^{A 3-/-}$ cells transfected with EGFP expression plasmid. (b) Comparison of numbers of genome-wide indels in WT 293FT and 293FT ${ }^{A 3-/-}$ cells transfected with EGFP expression plasmid. Means \pm s.d. were from four independent colonies. $P$ value, one-tailed Student's $t$ test. 


\section{a}

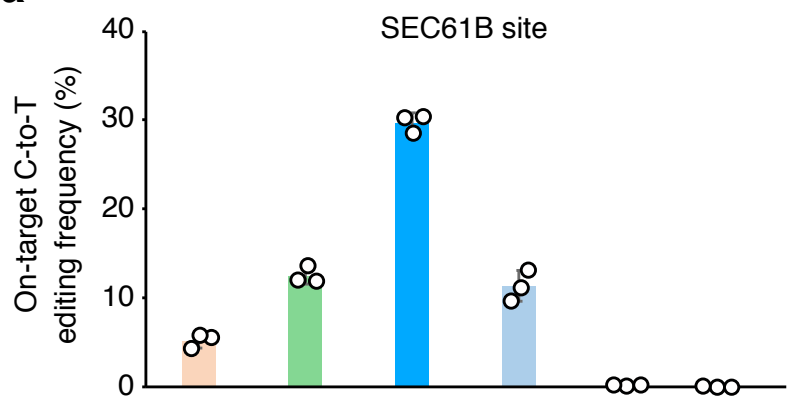

PE3 + pegRNA + nicking sgRNA (-73)

PE3 + pegRNA + nicking sgRNA (-29)

PE3 + pegRNA + nicking sgRNA (+53)

PE3 + pegRNA + nicking sgRNA (+103)

Cas9 + sgRNA

EGFP

b

RNF2 site

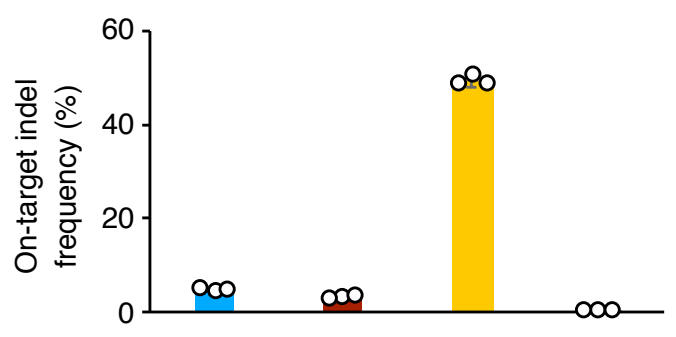

PE3 + pegRNA + nicking sgRNA

hA3A-BE3 + sgRNA

Cas9 + sgRNA

EGFP
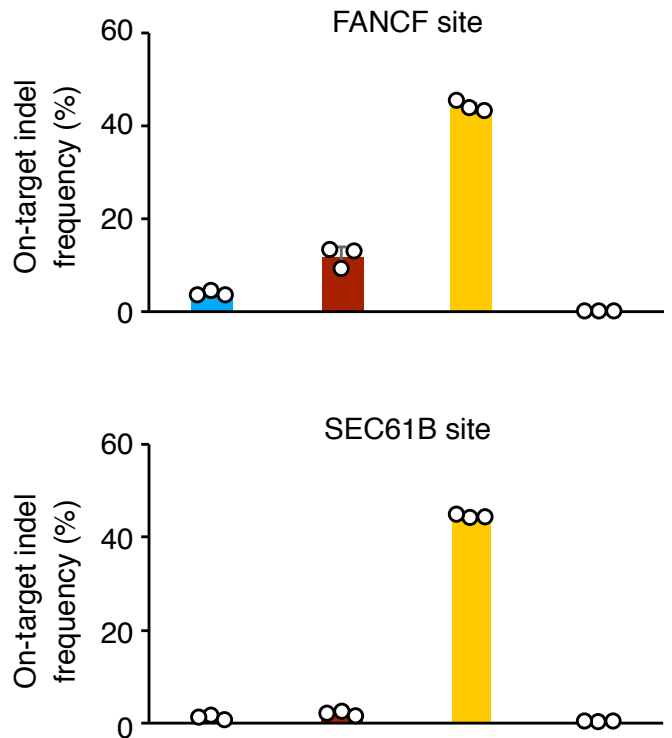

Supplementary Fig. 3. Optimization of nicking gRNA for generating targeted

base substitutions at $S E C 61 B$ site and unintended on-target indel frequency.

(a) On-target C-to-T editing frequencies induced by PE3, pegRNA and different

nicking positions, with Cas9 and EGFP as control. (b) On-target unintended indel 
bioRxiv preprint doi: https://doi.org/10.1101/2021.04.09.439109; this version posted April 9, 2021. The copyright holder for this preprint

(which was not certified by peer review) is the author/funder. All rights reserved. No reuse allowed without permission.

frequencies induced by PE3, hA3A-BE3, Cas9 and EGFP. Means \pm s.d. were from three independent colonies. 
a

EGFP-1 EGFP-1

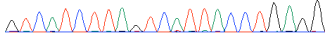
PE3-1

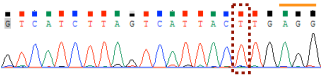
hA3A-BE3-1

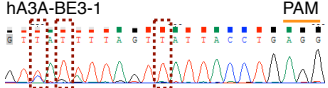

Cas9-1

CT deletion

Cas9-1 TPAM

WWMAMWWMMWh

b

EGFP-1 PE3-1

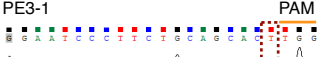
MWWWMWhWm: hA3A-BE3-1

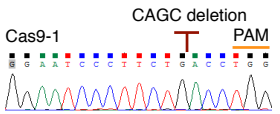

\section{C} EGFP-1 1 whonhownmowh

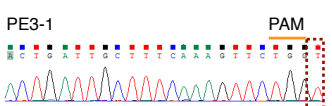
hA3A-BE3-1

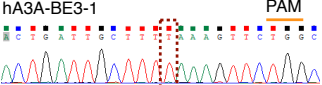
Cas9-1 $T$ insertion .

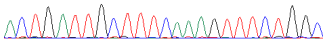

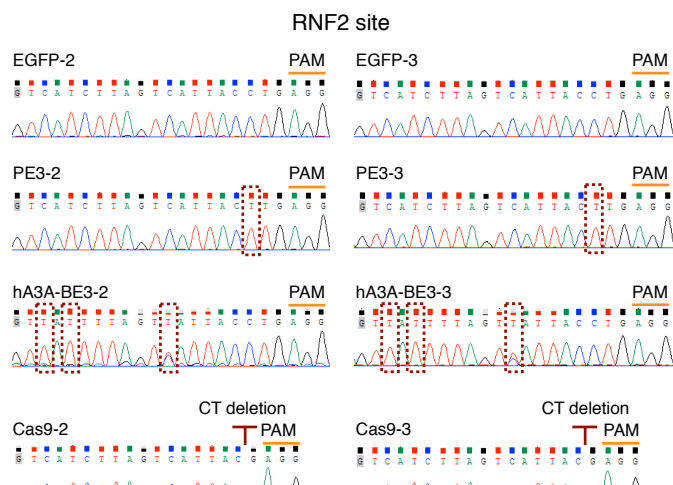

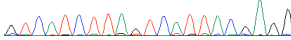

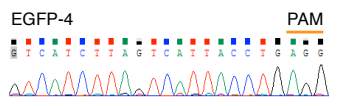

PE3-4

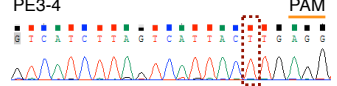

hA3A-BE3-4
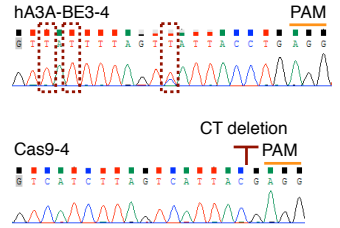

mWMManmowh

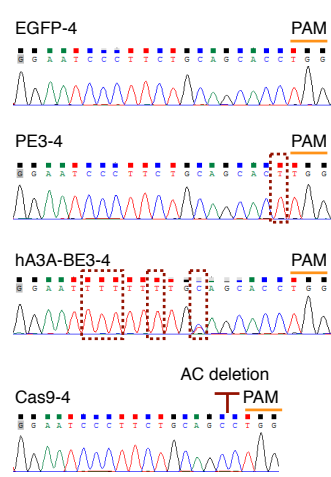

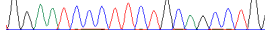

FANCF site

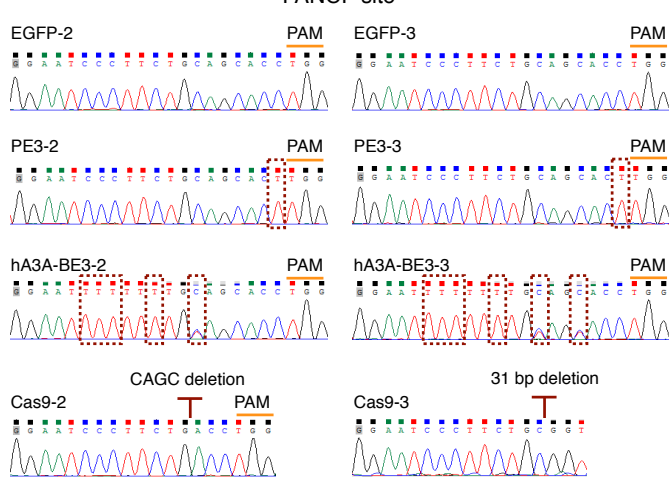

\section{Supplementary Fig. 4. Sanger sequencing of on-target sites in single cell colonies}

\section{containing intended base substitutions.}

Sanger sequencing of on-target sites in single cell colonies treated with EGFP, PE3,

hA3A-BE3 and Cas9 at RNF2 (a), FANCF (b) and SEC61B (c) target sites. 
a

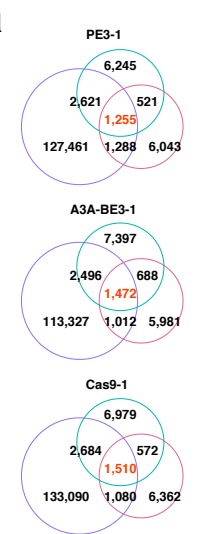

b

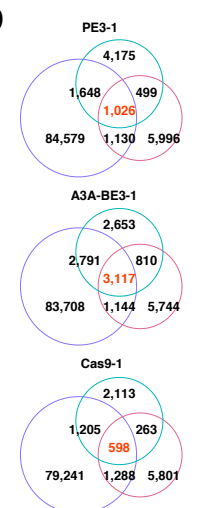

C

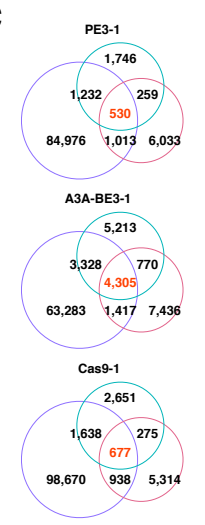

d

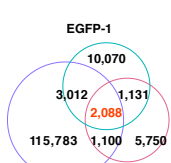

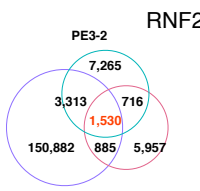

RNF2 site
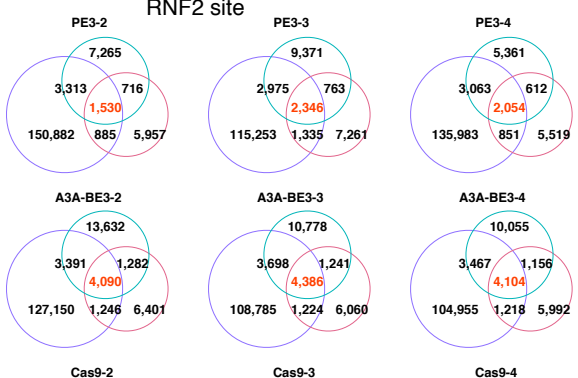

Cas9-2
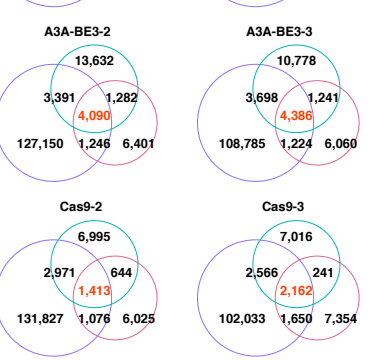

(1)

A3A-BE3-4

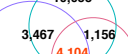

$\begin{array}{lll}104,955 & 1,218 & 5,992\end{array}$

Cas9-4

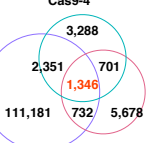

FANCF site
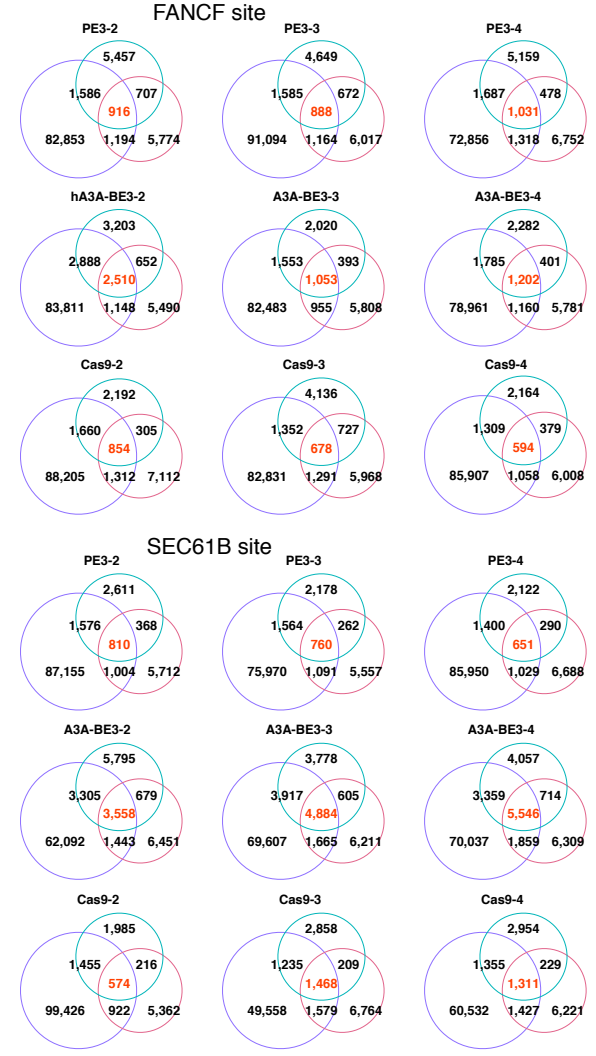

A3A-BE3-3

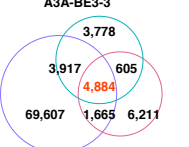

A3A-BE3-4

4,057

3,359 714

$\begin{array}{lll}70,037 & 1,859 & 6,309\end{array}$

Cas9-4

2954

1,355 229

$\begin{array}{lll}60,532 & 1,427 & 6,221\end{array}$

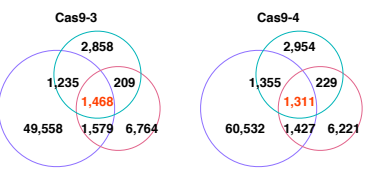

EGFP transfection
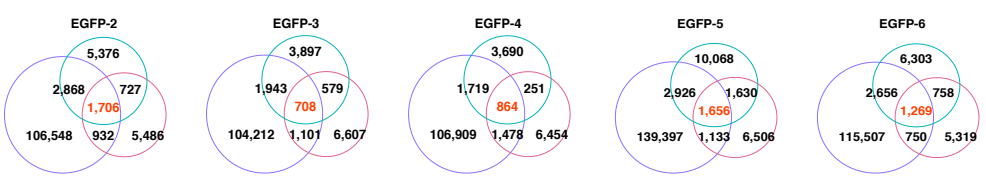

\section{Supplementary Fig. 5. Venn diagrams of genome-wide base substitutions}

\section{induced by genome editors when generating targeted base substitutions.}

Venn diagrams of genome-wide base substitutions in single cell colonies detected by three callers after PE3, hA3A-BE3 and Cas9 treatment at RNF2 (a), FANCF (b) and 
SEC61B (c) target sites. (d) Venn diagrams of genome-wide base substitutions detected by three callers in EGFP treated single cell colonies. 
a

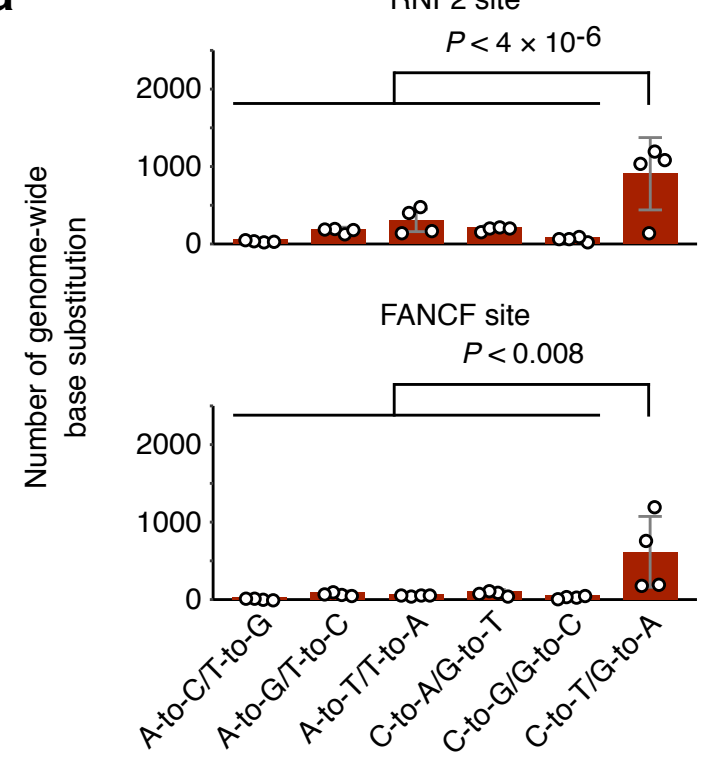

b

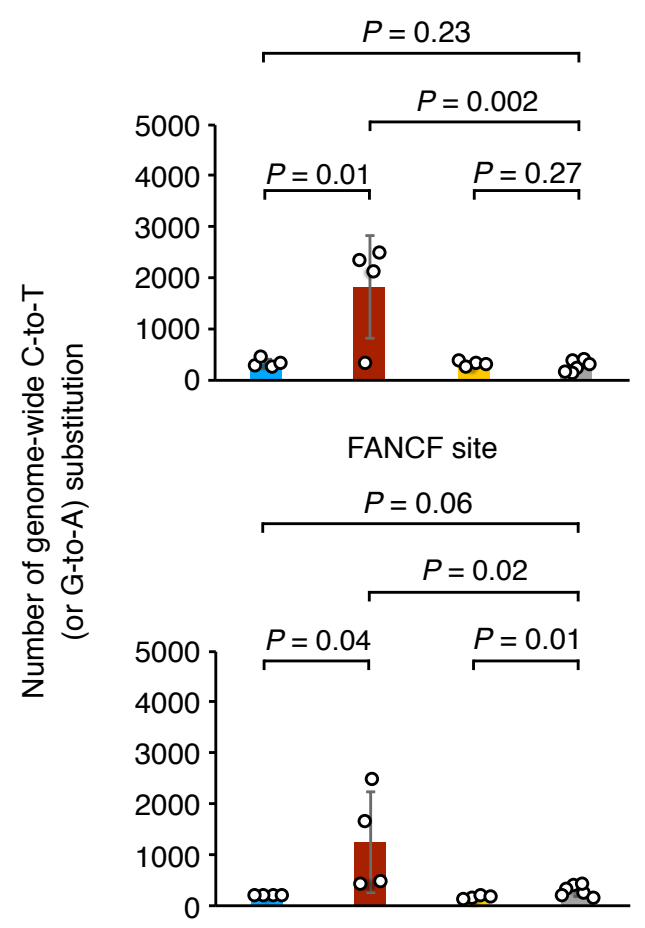

hA3A-BE3 + sgRNA

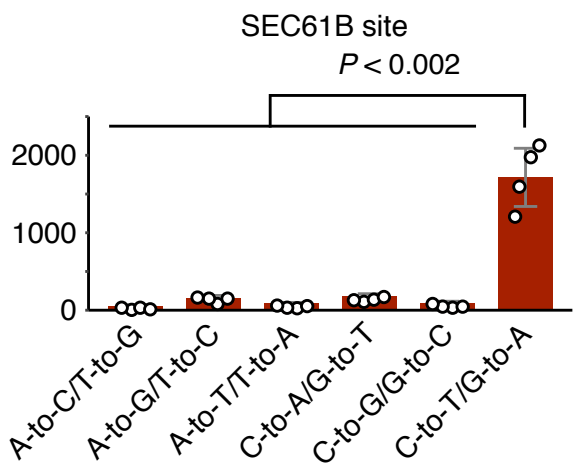

PE3 + pegRNA + nicking sgRNA

hA3A-BE3 + sgRNA

Cas9 + sgRNA

EGFP

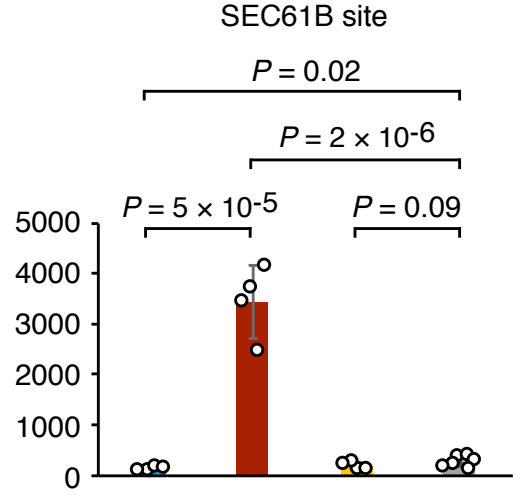

Supplementary Fig. 6. hA3A-BE3 induced genome-wide C-to-T or A-to-G mutations.

(a) Histograms to show numbers of all six types of genome-wide base substitutions from cells treated with hA3A-BE3. (b) Numbers of genome-wide C-to-T/A-to-G 
substitutions from cells treated with EGFP, Cas9, hA3A-BE3 and PE3. Means \pm s.d. were from four independent colonies. 
bioRxiv preprint doi: https://doi.org/10.1101/2021.04.09.439109; this version posted April 9, 2021. The copyright holder for this preprint (which was not certified by peer review) is the author/funder. All rights reserved. No reuse allowed without permission.

a

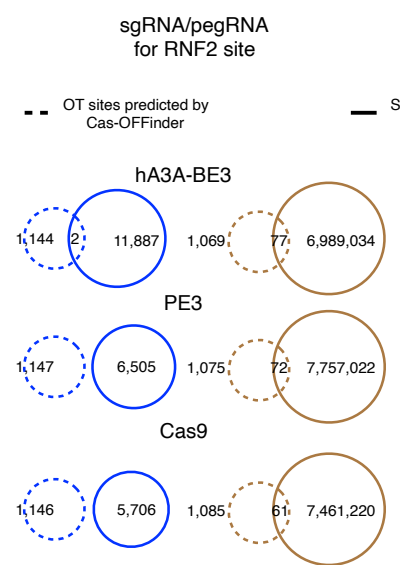

Cas-OFFinder

GTCATCTTAGTCATTACCTGAGG On-targe

-GT----A------------T-- chr5:92701253-92701275

-----C----GC----------- chr8:755960-755982

chr10:129047187-129047209

-----C--------TA---G-- chr17:17168755-17168777

hA3A-BE3 (WGS identified)

GTCATCTTAGTCATTACCTGAGG On-target

CAGC---GCA-AT-GC-GCT--- chrs:113237089

CATCGGC-TCCATA-CTTCC--- chr2:240596936

CC-TCCCD-CCC

$-G-C-C C-P C-C C G-T G C---$ chr1 21865340

PE3 (WGS identified)

GTCATCTTAGTCATTACCTGAGG On-target

C--TAGCCT-GACCCT--CTTCC chr22:48531624

-AGGCT-ATI-GGGC-G--CCCT chr5:15300329

TGT-OT-AT-CT-A-TTGCCTA- chr6:15000

AAA--AA-TT-GG--CA--TCAT chr1:15372642

Cas9 (WGS identified)

GTCATCTTAGTCATTACCTGAGg On-target

C-T-AT--TC-TGGGTAT-CTTIT chr2:137335952

CAAGCA-A-AAATA-TTTCTGAT chr6:45201060

C-GCC--GG-CGC-C-A-ATG-- Chr1:91547100

ACAGAAGA-A-GC-A-GTCCT-C chr1:99618970

d

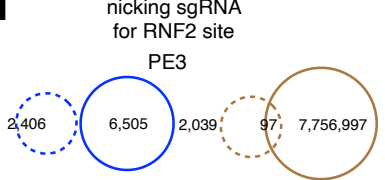

Cas-OFFinder

TCAACCATTAAGCAAAACATGGG On-targe

- Com-OFFinder

--------A---CT--------T-- chr12:118440560-11844058

-

chr5:2009783-2009806

PE3 (WGS identified)

TCAACCATTAAGCAAAACATGGG On-target

-G-GG-CACCTC--TCT-CA-CT chr5:19333612

C-СТT-CC--C-TCСТT-ССТCC chr17:21293360

-AT-TI-ATCCTI-G-TI-CTA Chr2.198400010

AT--AATG-TTTIT-TTT--ACT Chr5.7270009 b

sgRNA/pegRNA
for FANCF site

SNVs called from WGS
of 4 colonies

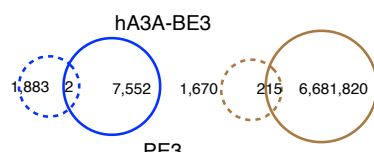

$\underbrace{3,398}_{\text {Cas9 }}$

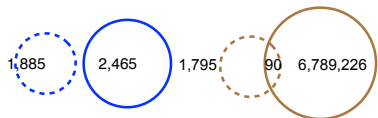

Cas-OFFinder

GGAATCCCTTCTGCAGCACCTGG On-target

---G-----C--A-------A-- chr10:37664254-37664277

A--GG---C------------A-- chr17:80950161-8095018

T-------A---C--------A-- chr10:71703361-71703384

hA3A-BE3 (WGS identified)

GGAATCCCTTCTGCAGCACCTGG On-target

TTTGA-AGAC--CAGTGG----T chr19:1648725

ATG-ATT-AAA-ATATT-ACAT chr10:90768746

T-T--G-АССТ-САТT-T-TGAA chr15:37768967

TCTT--TTC-T--АТ-TCT--TT chr9:113955813

PE3 (WGS identified)

GGAATCCCTTCTGCAGCACCTGG On-target

--GGAT-T-CT--A-TAGGGCCA chr1:34629125

ATCGAGG-AC--C---ACGA-OT chr7:154901036

-ATGA-AAAA---AGTGT-TAA- chr17:16942227

TTT-GGATAGAACT-AGGTTG-- chr15:73840642

ATGCAGGGACACTAG-GTT----- chr2:89181602

Cas9 (WGS identified)

GGAATCCCTTCTGCAGCACCTGG On-target

CA-GG-TT-AA---TTGGGGCTC chr11:113332411

AATGAA-TG-TCTATTTCTG--- chr3:77113625

ATCCAATTA-T-AAG--TG-TC chr2:2199290

A----GT--GTGATGTA-TA-CA chr5:45508695

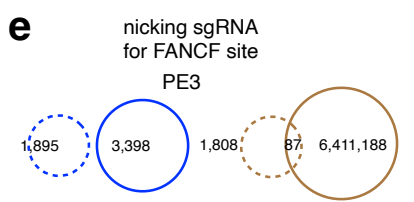

Cas-OFFinder

GGGGTCCCAGGTGCTGACGTAGG On-target

-------------------A-G--- chr17:55845703-55845726

-T---------------G--G-- chr:144566440-1445664

-----GG----------G--G-- chrr11:643889934-64388957

PE3 (WGS identified)

GGGGTCCCAGGTGCTGACGTAGG On-target

CTCAGAAG-TCCCAGCC-CGTCT chrl:18100748

CT-CCAGGT-CCC-A--GTATT- Chr3:188277360

T-I-GAAG-TG-AAAG-AGGAT Chr20.37657692

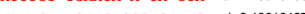

C

sgRNA/pegRNA

Union of raw SNVs by GATK,
Lofreq and Strelka2

hA3A-BE3

PE3

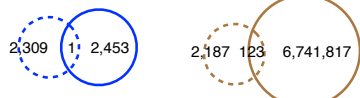

Cas9

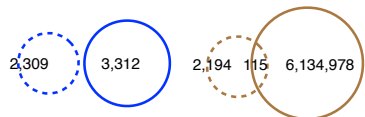

Cas-OFFinder

ACTGATTGCTTTCAAAGTTCTGG On-targe

--------G-T---A------- chr16:4727805-4727827

-----C---------T---A--- chr15:36978872-36978894 - chr15:91540267-91540289

hA3A-BE3 (WGS identified)

ACTGATTGCTTTCAAAGTTCTGG On-target

-- AAGCCAA-C---TGAAATCCT chr6:33951517

-AAC-C-C-AA-G-C-TCA-CTT chr: 134718169

GGCC-GA-AGGAG-GG-ACGCCC chr15:68500929

GAAGCCTTAC----G---TGCC chr8.133561088

PE3 (WGS identified)

ACTGATTGCTTTCAAAGTTCTGG On-target

CTGACAA-AGAAT-T-AA-A-T Chr15:42170619

-TCTTAA-G--C----A-G--TT chr6:169431901

-AGAT-AC-A-AGTC-C-AGAA- chr9:102827721

-GATTCCC--CC-TGCCCC-GCT chr12:280455

TGCATGA-TGCA-T--AAG-ACT chr8:138265571

Cas9 (WGS identified)

ACTGATTGCTTTCAAAGTTCTGG On-target

-AA-AA-TCC--C--AC-GAAC chr22:33841950

G-CATA--GAAAGTT-CA-A-A- chr1:41276346

GAATC-AT--AAG-C-AA-AGAA chr3:97798102

G--CCA-AAACAAT-TT--AAAA chr7:8351651

GTACTGAATG--TTGCTC-G-CT chr5:13756468

f nicking sgRnA

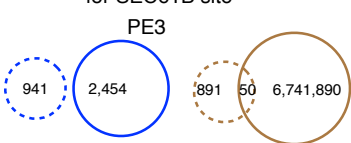

Cas-OFFinder

GCCCTGCTACATGACGGAGTGGG On-target

-----A------G-A----T-- chr1:51605016-51605039

--------------AT---AA-- chr4:145753910-145753933

C-G--------------TC-T-- chr1 10:62123833-621240

PE3 (WGS identified)

GCCCTGCTACATGACGGAGTGGG On-target

TTAT-TA-T-C-A-TTT-ACAT- chr7:96912096

T-TT-A---GCAACATC-TAA-A chr4:161153895

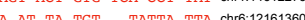

A-AT-TA-TGI---TATA-THA CHF 121613005

Supplementary Fig. 7. Few overlap of OT sites predicted by Cas-OFFinder and

identified by WGS. 
(a-c) Comparison of the OT sites predicted by Cas-OFFinder based on pegRNA spacer regions for $R N F 2, F A N C F$ and $S E C 61 B$ sites and the OT sites identified by WGS. (d-f) Comparison of the OT sites predicted by Cas-OFFinder based on the nicking gRNA spacer regions for $R N F 2, F A N C F$ and $S E C 61 B$ sites and the OT sites identified by WGS. The on-target sequences were in black and the mismatched bases at OT sites were in red. The intersection (blue circle) or union (brown circle) of callers were used to identify SNVs from WGS data. 
a ANK2

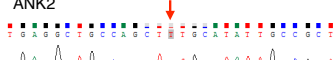
MWhWhwhommonm CAPN10 Mrommomhanowhowly CELSR2

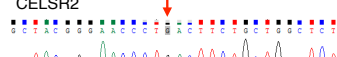

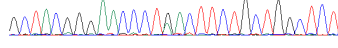

EEPD1

WhWhWmWh

HSPG2

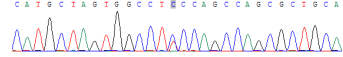

NIF3L1

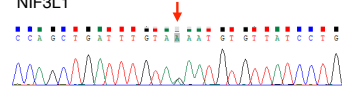

RASSF1

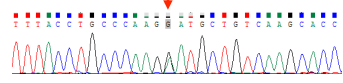

TG

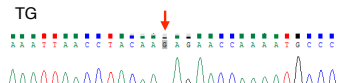

TMEM181

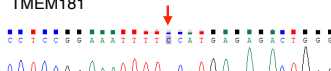

MmomphwawhWWhly

vav1

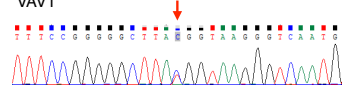

b

ANK2

An

CAPN10

antang

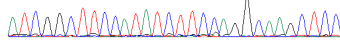

CELSR2

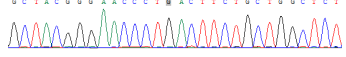

EEPD1

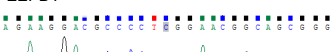

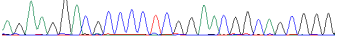

HSPG2

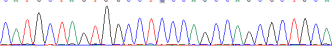

NIF3L1

Mandonmowhlwhmon

RASSF1

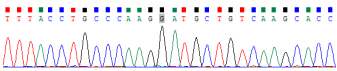

TG

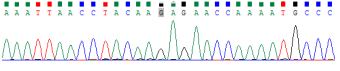

TMEM181

and:

VAV 1

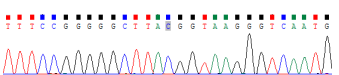

Supplementary Fig. 8. Sanger sequencing of OT site identified by WGS in single cell colonies.

Sanger sequencing of OT site identified by WGS in single cell colonies treated with hA3A-BE3 and RNF2-targeting gRNA (a) or EGFP (b). 
a

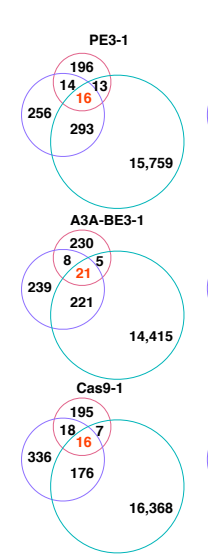

b

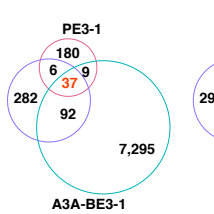

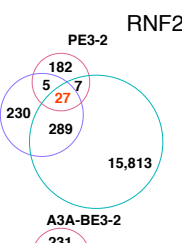

RNF2 site
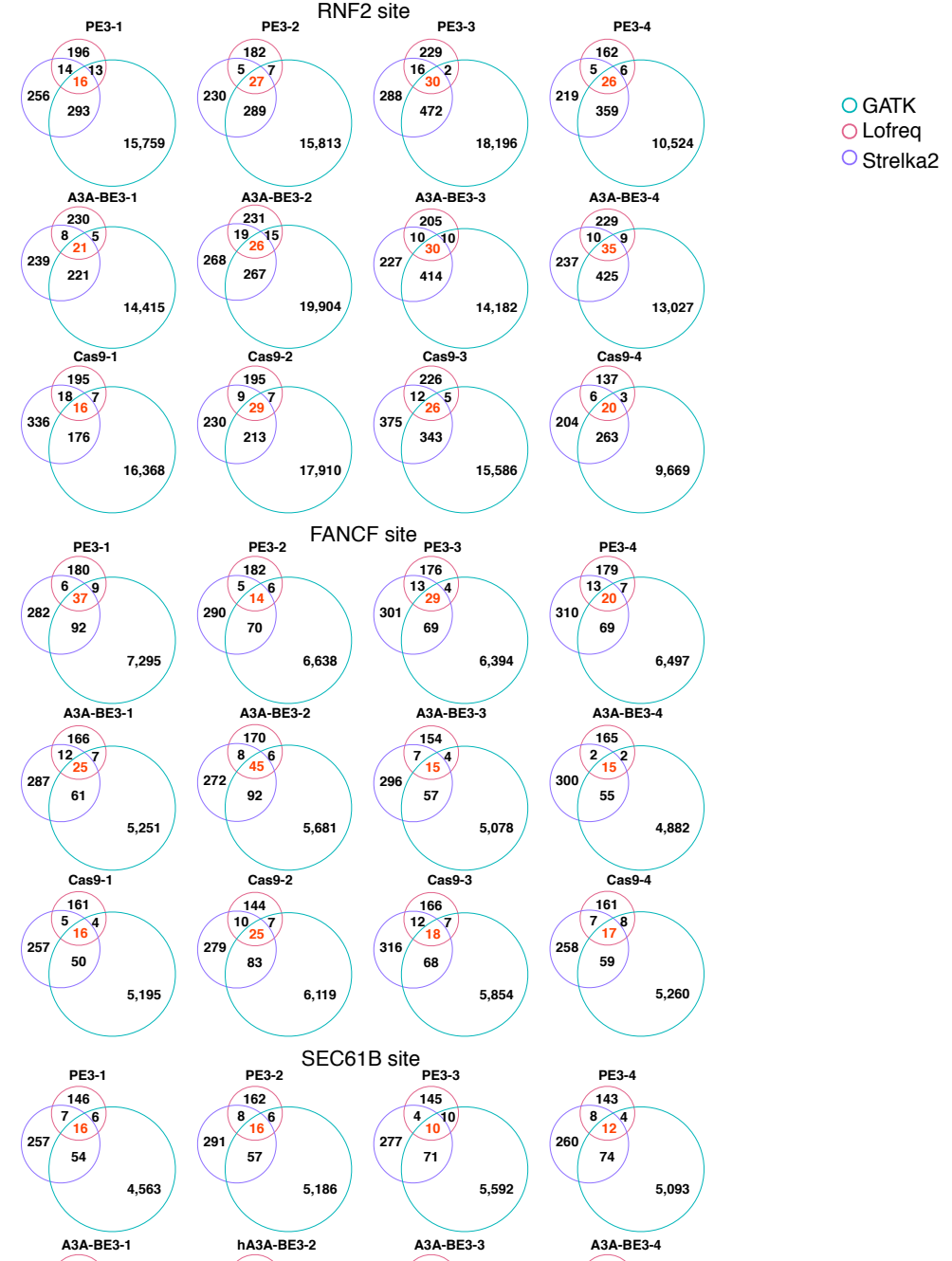

A3A-BE3-4

Strelka2
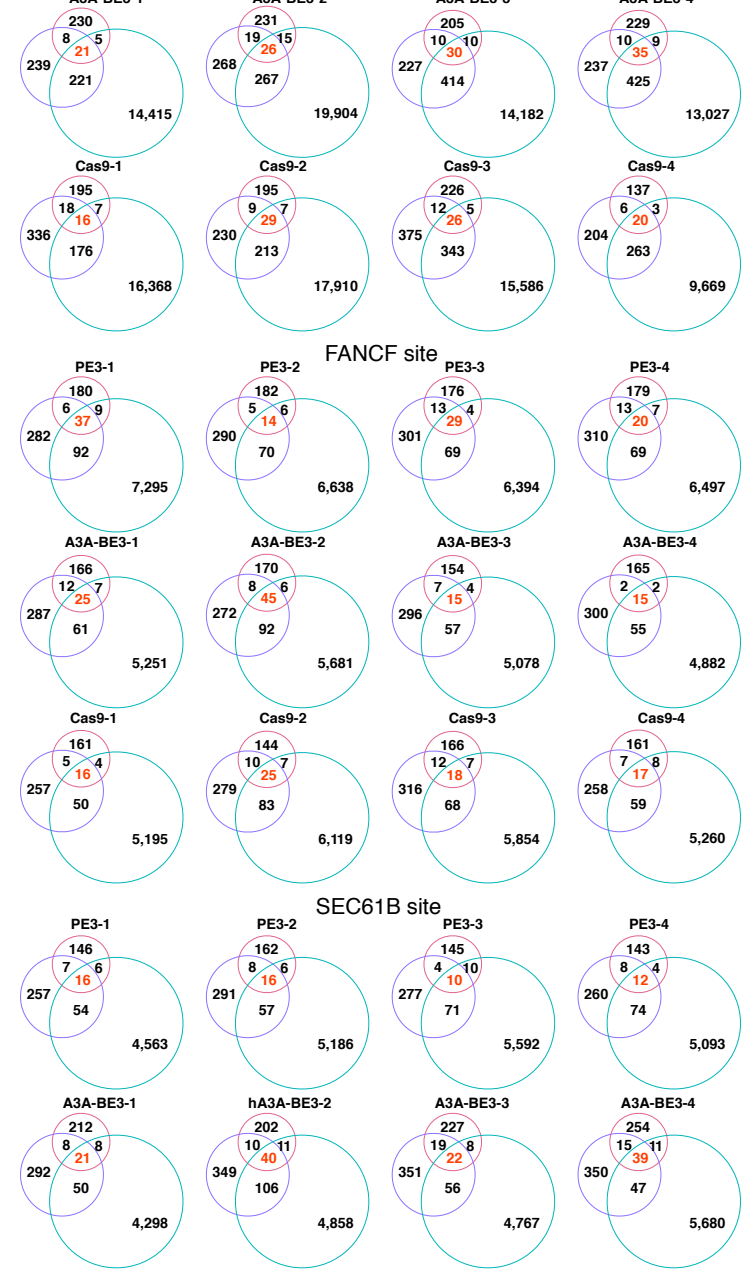

A3A-BE3-4
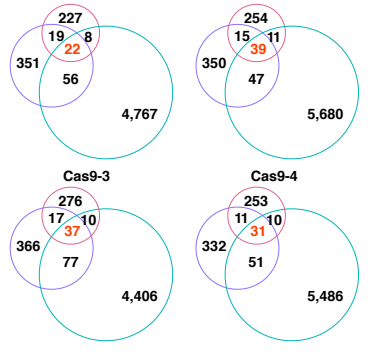

d
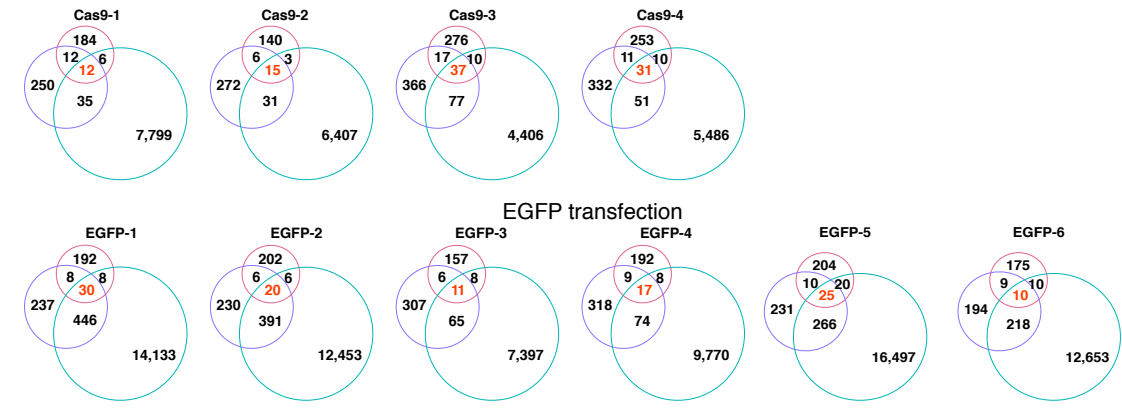

Supplementary Fig. 9. Venn diagrams of genome-wide indels induced by genome editors when generating targeted base substitutions.

(a) Venn diagrams of genome-wide indels in single cell colonies detected by three callers after PE3, hA3A-BE3 and Cas9 treatment at RNF2 (a), FANCF (b) and 
SEC61B (c) target sites when generating targeted base substitutions. (d) Venn diagrams of genome-wide indels detected by three callers in EGFP treated single cell colonies. 
a

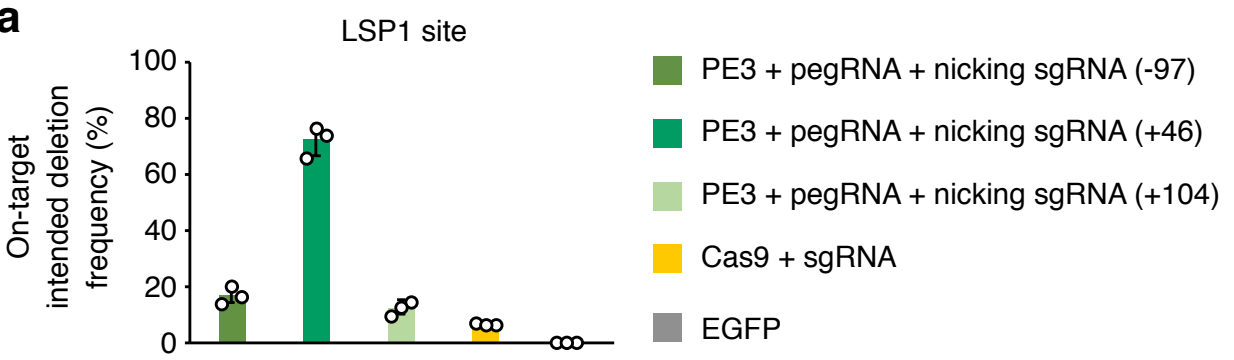

b

EMX1 site

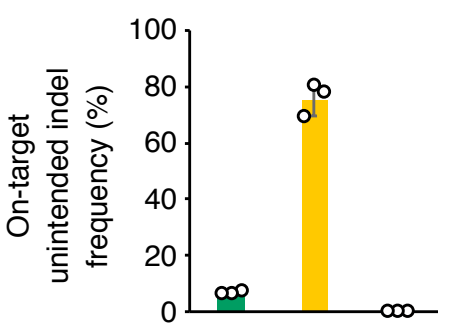

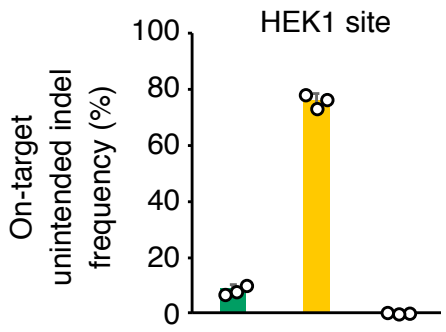

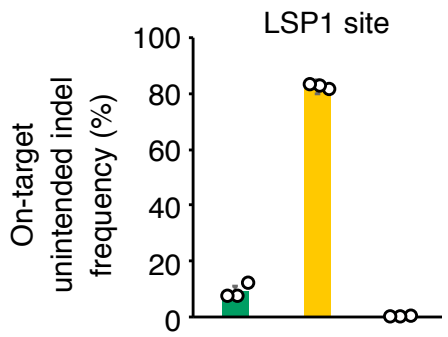

Supplementary Fig. 10. Optimization of nicking gRNA for generating targeted

deletions at $L S P 1$ site and unintended on-target indel frequency.

(a) On-target intended deletion frequencies induced by PE3, pegRNA and different nicking positions, with Cas9 and EGFP as control. (b) On-target unintended indel 
bioRxiv preprint doi: https://doi.org/10.1101/2021.04.09.439109; this version posted April 9, 2021. The copyright holder for this preprint

(which was not certified by peer review) is the author/funder. All rights reserved. No reuse allowed without permission.

frequencies by PE3, Cas9 and EGFP. Means \pm s.d. were from four independent colonies. 
a

EMX1 site

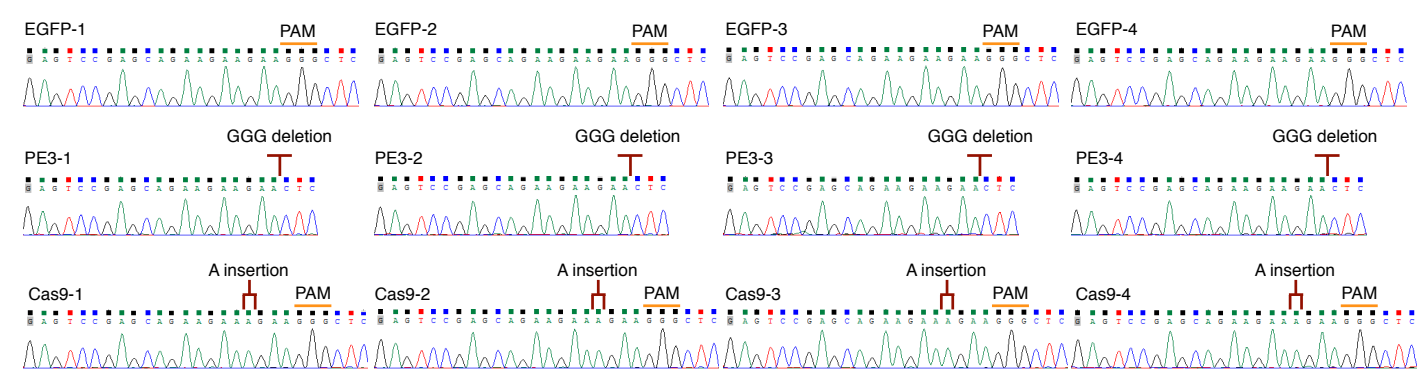

b

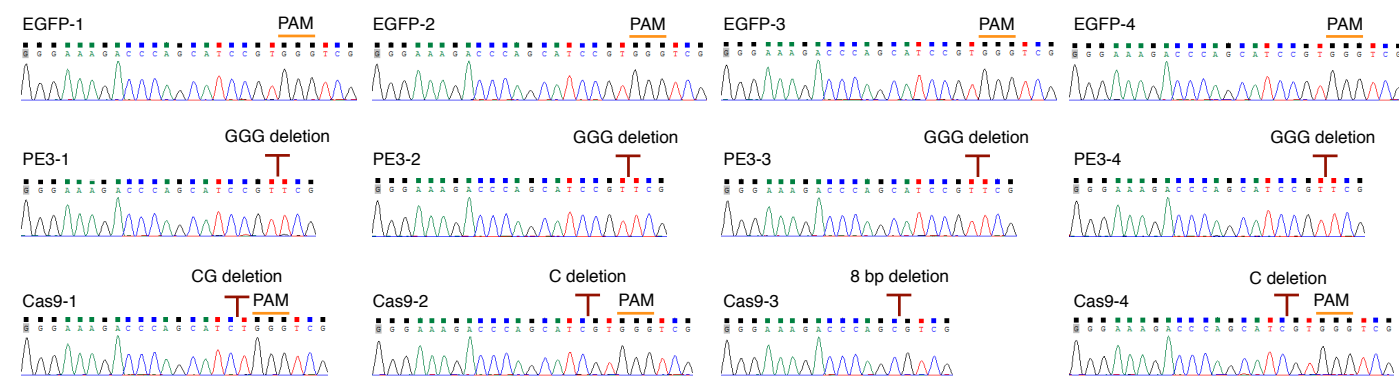

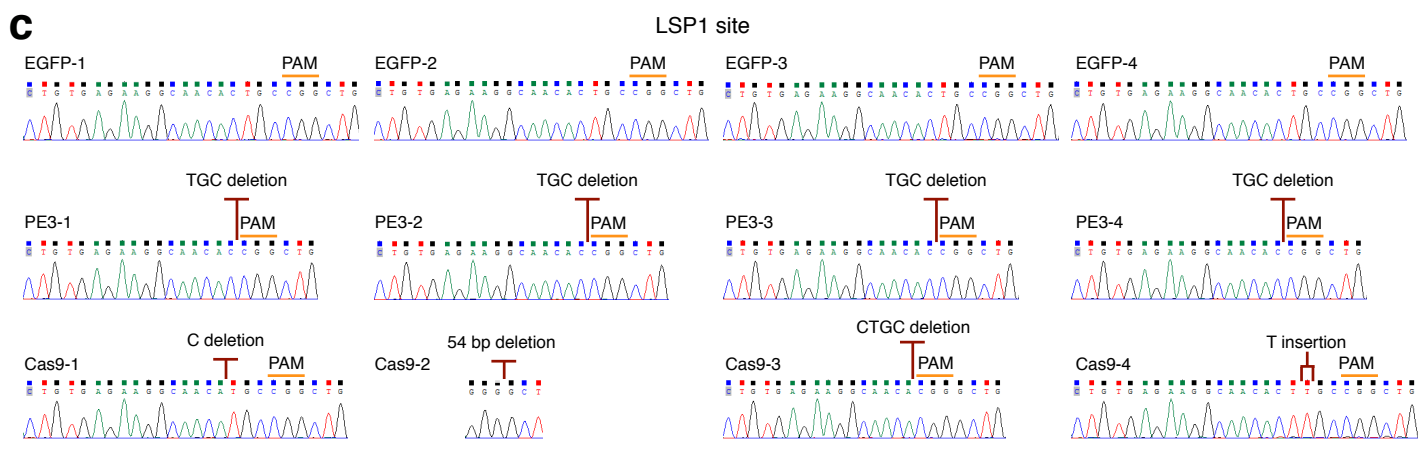

Supplementary Fig. 11. Sanger sequencing of on-target sites in single cell

colonies containing intended deletions.

Sanger sequencing of on-target sites in single cell colonies after EGFP, PE3, Cas9

treatment at EMXI (a), HEKI (b), LSPI (c) target sites when generating intended deletions. 


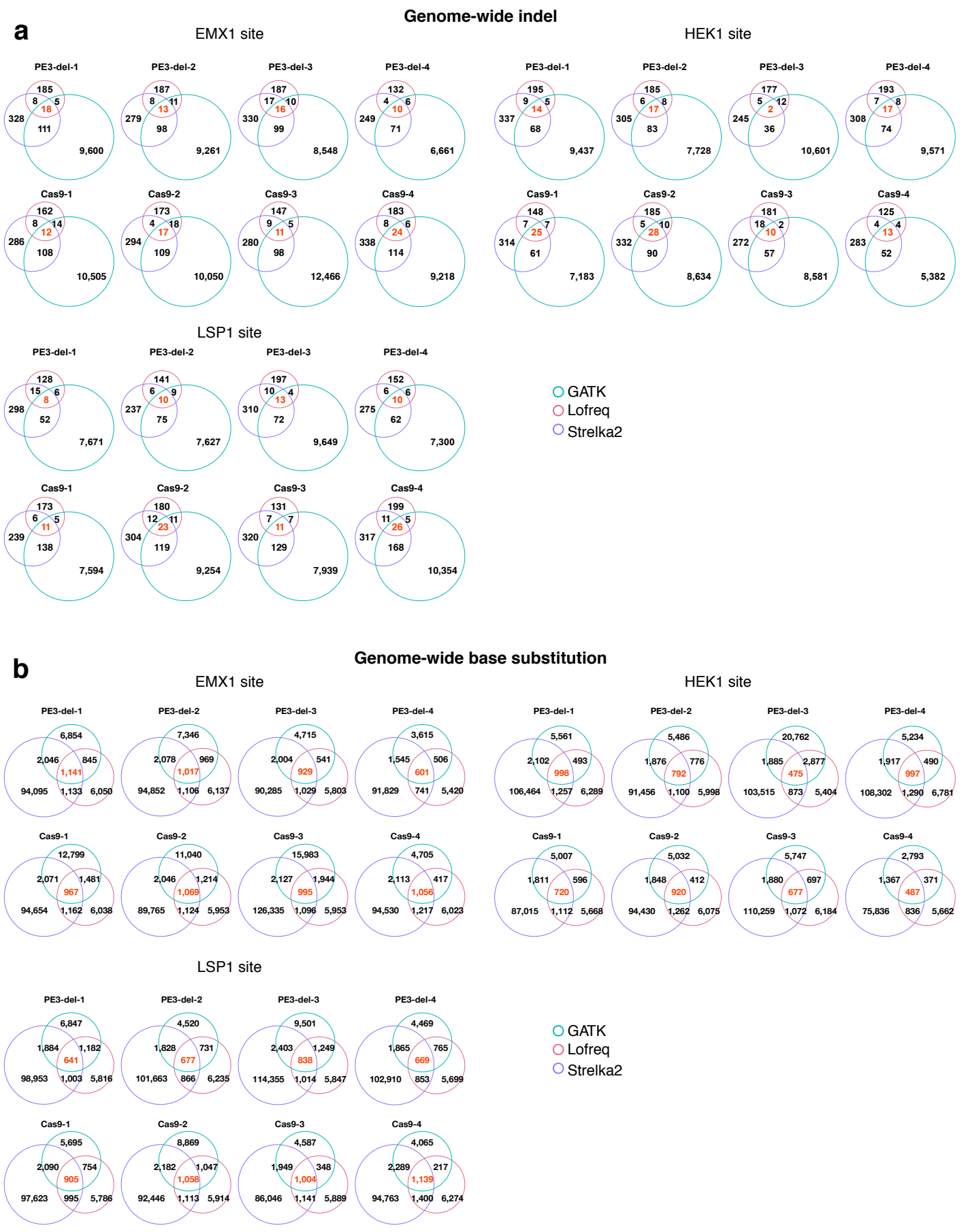

Supplementary Fig. 12. Venn diagrams of genome-wide indels and base

substitutions induced by genome editors when generating targeted deletions.

(a) Venn diagrams of genome-wide indels in single cell colonies detected by three callers after PE3 and Cas9 treatment at EMX1, HEK1, LSP1 target sites when 
generating targeted deletions. (b) Venn diagrams of genome-wide base substitutions in single cell colonies detected by three callers after PE3 and Cas9 treatment at EMX1, HEK1, LSP1 target sites when generating targeted deletions. 
a

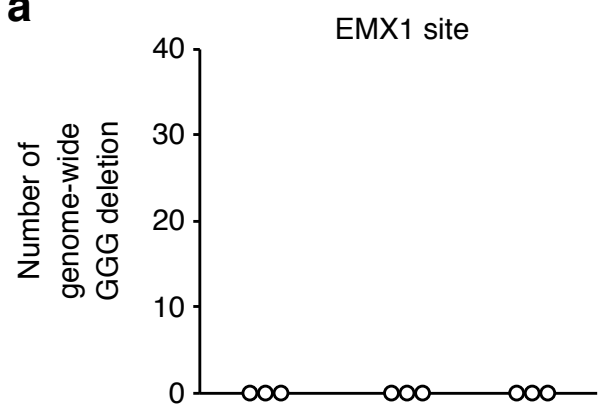

b
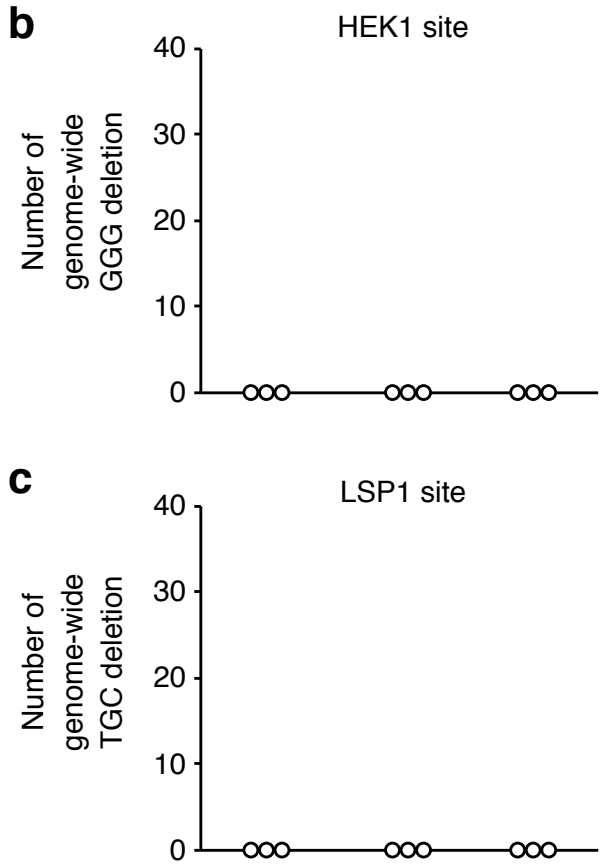

PE3 + pegRNA + nicking sgRNA

Cas $9+$ sgRNA

EGFP

\section{Supplementary Fig. 13. PE3 induced no intended 3-bp deletion on a genome-}

\section{wide scale.}

(a) Numbers of genome-wide GGG deletions after cells treated with PE3, Cas9 and

EGFP at EMX1 site when generating targeted GGG-deletion. (b) Numbers of

genome-wide GGG deletions after cells with PE3, Cas9 and EGFP at HEK1 site when

generating targeted GGG-deletion. (c) Numbers of genome-wide TGC deletions after

cells treated with PE3, Cas9 and EGFP at $L S P 1$ site when generating targeted TGC

deletions. 


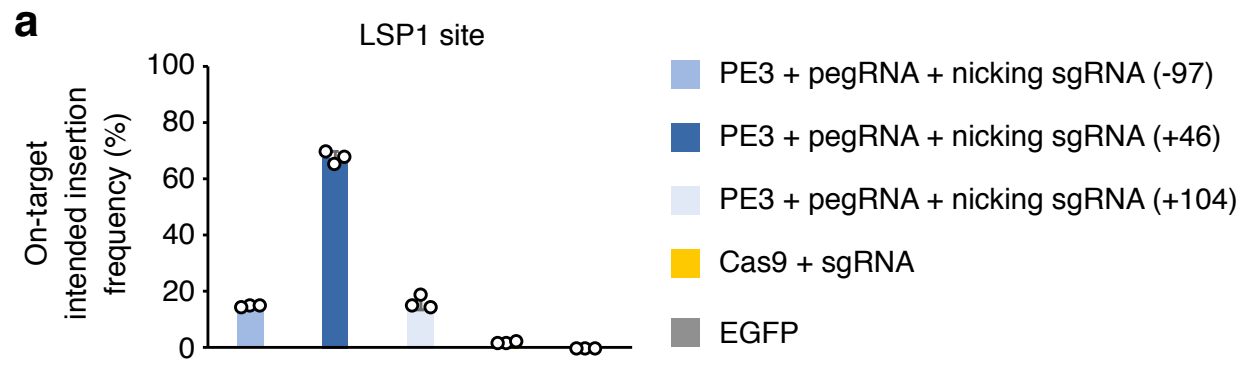

b EMX1 site

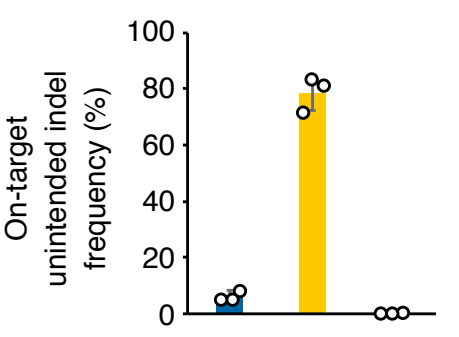
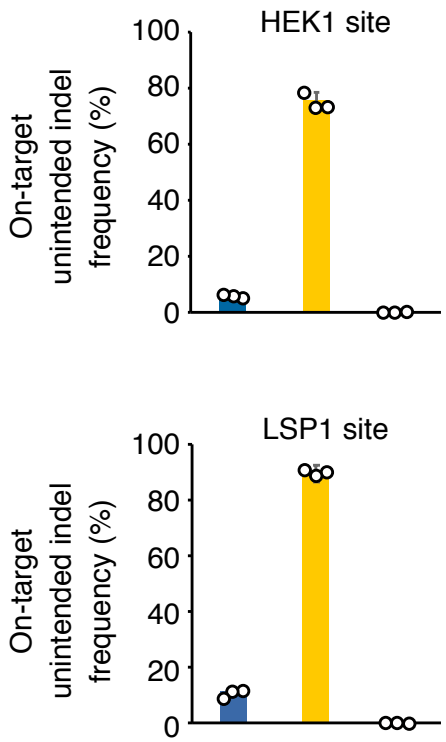

\section{Supplementary Fig. 14. Optimization of nicking gRNA for generating targeted} insertions at LSP1 site and unintended on-target indel frequency.

(a) On-target intended insertion frequencies induced by PE3, pegRNA and different nicking positions, with Cas9 and EGFP as control. (b) On-target unintended indel frequencies by PE3, Cas9 and EGFP at three target sites. Means \pm s.d. were from four independent colonies. 
a

EMX1 site

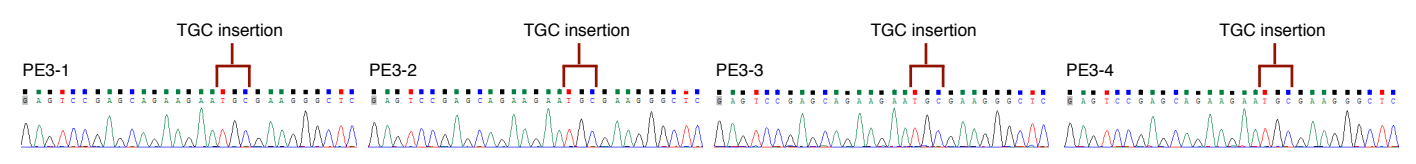

b

HEK1 site

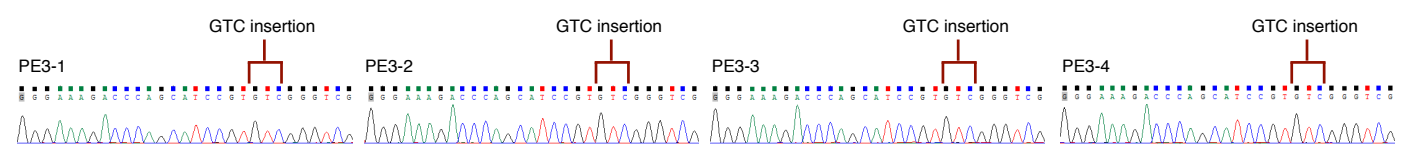

C

LSP1 site

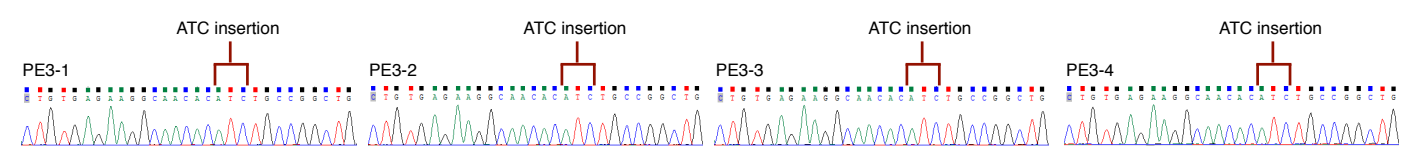

\section{Supplementary Fig. 15. Sanger sequencing of on-target sites in single cell}

\section{colonies containing intended insertions.}

(a) Sanger sequencing validation of on-target site in single cell colonies containing

intended TGC insertions after PE3 treatment at EMX1 site. (b) Sanger sequencing

validation of on-target site in single cell colonies containing intended GTC insertions

after PE3 treatment at HEK1 site. (c) Sanger sequencing validation of on-target site in single cell colonies containing intended ATC insertions after PE3 treatment at LSP1

site. 
a

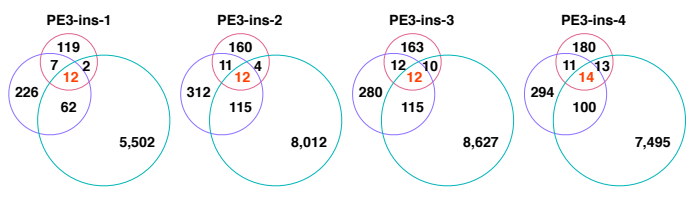

HEK1 site

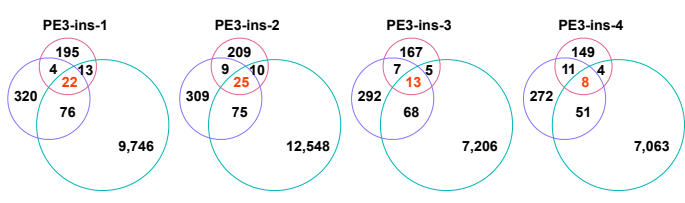

LSP1 site

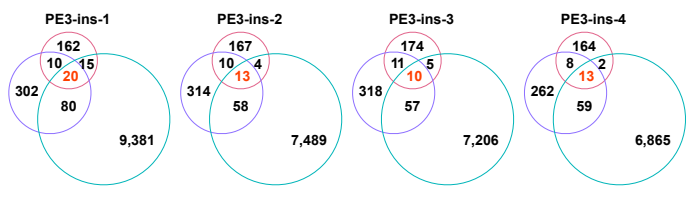

b Genome-wide base substitution EMX1 site

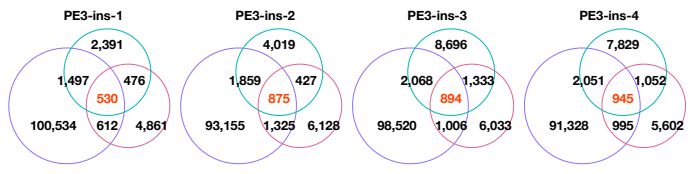

HEK1 site

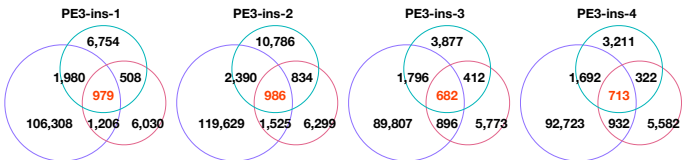

LSP1 site

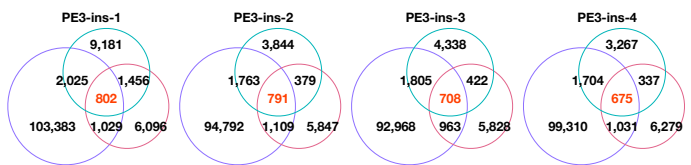

\section{Supplementary Fig. 16. Venn diagrams of genome-wide indels and base}

substitutions induced by genome editors when generating targeted insertions.

(a) Venn diagrams of genome-wide indels by three callers in single cell colonies detected after PE3 treatment at EMX1, HEK1, LSP1 sites when generating targeted insertions. (b) Venn diagrams of genome-wide base substitutions in single cell 
bioRxiv preprint doi: https://doi.org/10.1101/2021.04.09.439109; this version posted April 9, 2021. The copyright holder for this preprint (which was not certified by peer review) is the author/funder. All rights reserved. No reuse allowed without permission.

colonies detected by three callers after PE3 treatment at EMX1, HEK1, LSP1 sites when generating targeted insertions. 
a
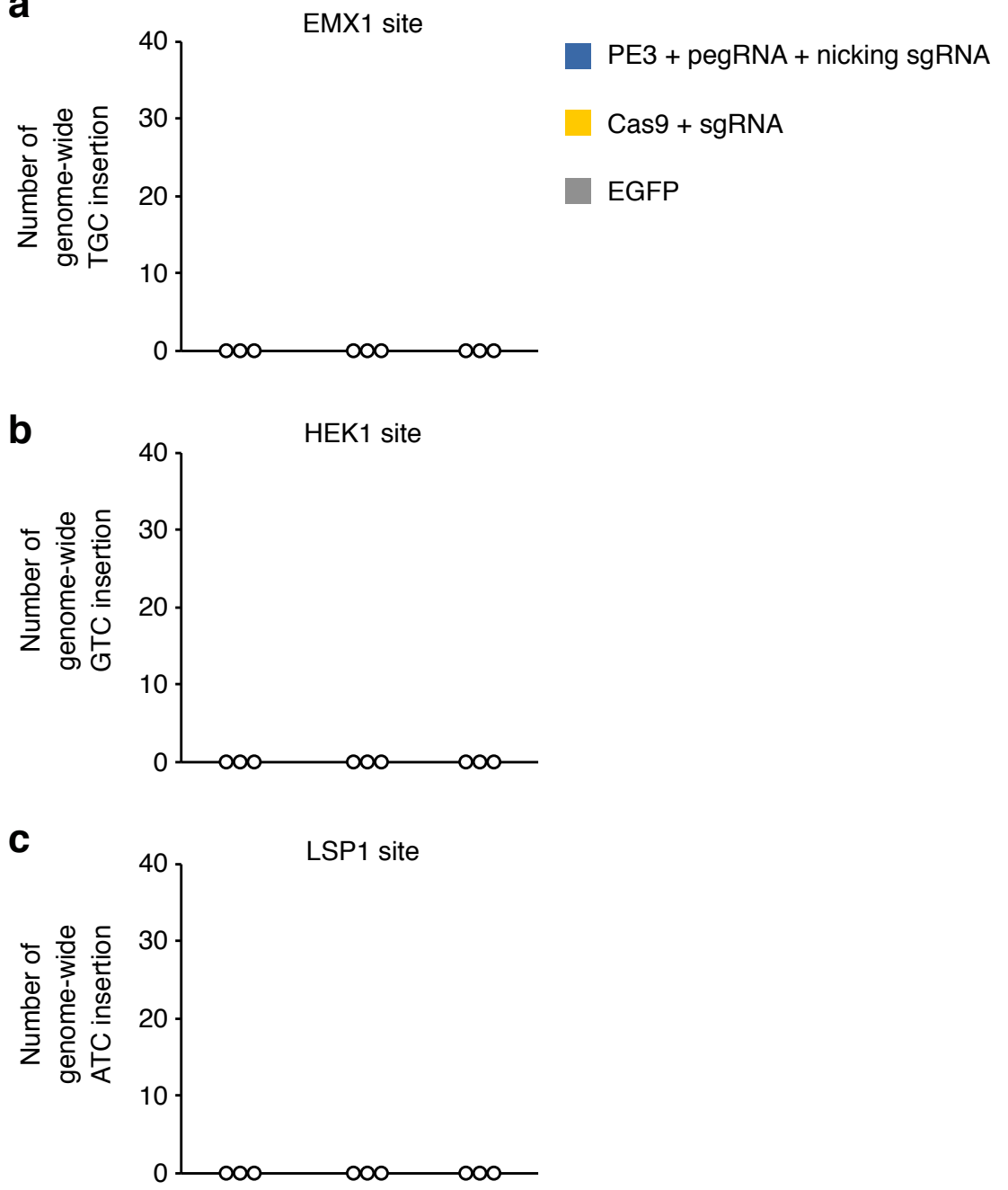

Supplementary Fig. 17. PE3 induced no intended 3-bp insertion on a genome-

wide scale.

(a) Numbers of genome-wide TGC insertions after cells treated with PE3, Cas9 and EGFP at EMX1 site. (b) Numbers of genome-wide GTC insertions after cells treated with PE3, Cas9 and EGFP at HEK1 site. (c) Numbers of genome-wide ATC insertions after cells treated with PE3, Cas9 and EGFP at LSP1 site. 
a

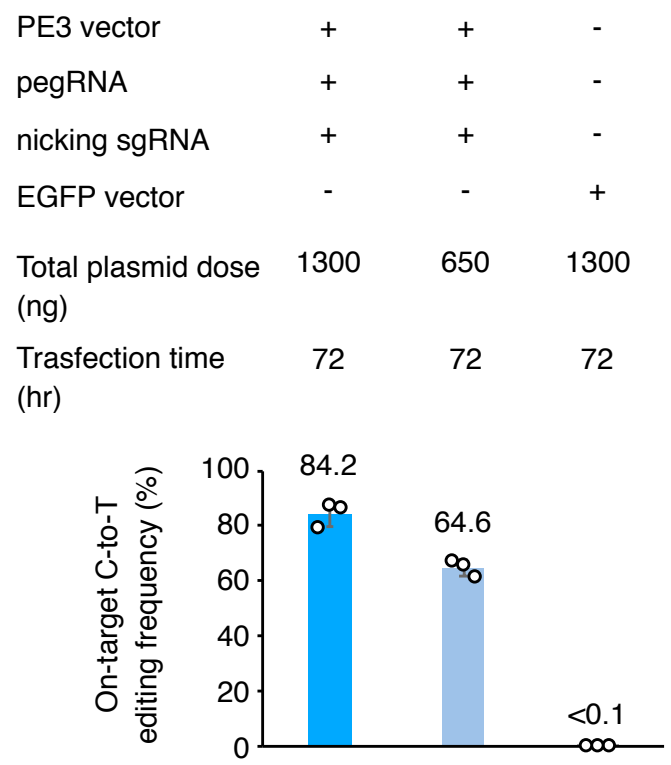

b

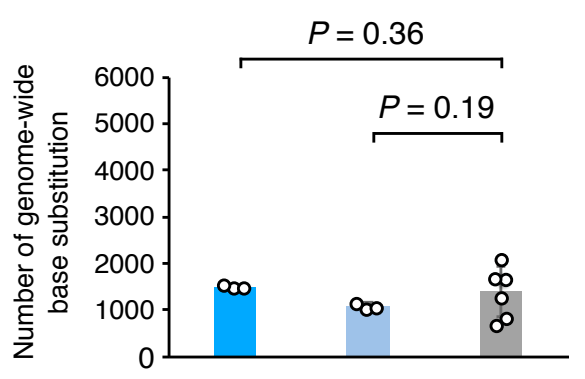

C

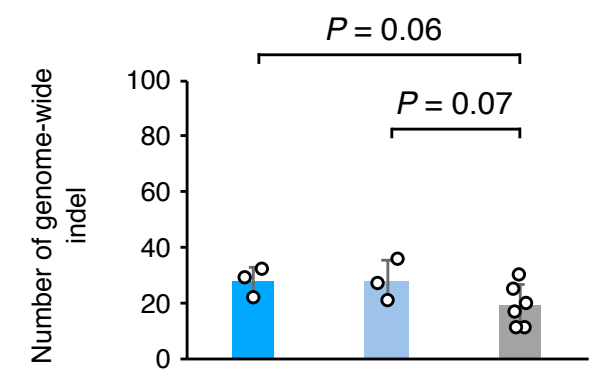

RNF2 site
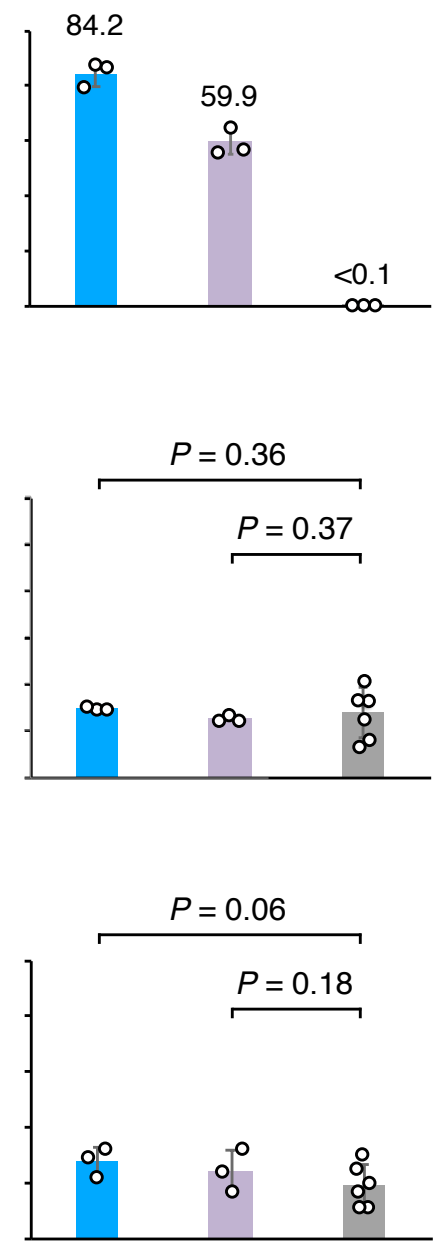

Supplementary Fig. 18. PE3 induced background levels of genome-wide OT mutations via plasmid delivery of different doses and time periods.

(a) On-target C-to-T editing frequencies induced by PE3 via plasmid delivery of different doses and time periods. (b) Numbers of genome-wide base substitutions 
induced by PE3 via plasmid delivery of different doses and time periods. (c) Numbers of genome-wide indels induced by PE3 via plasmid delivery of different doses and time periods. The data for Cas9 and EGFP in (a-c) are the same. 
a

Pipeline to examine reads that contain potential pegRNA sequence

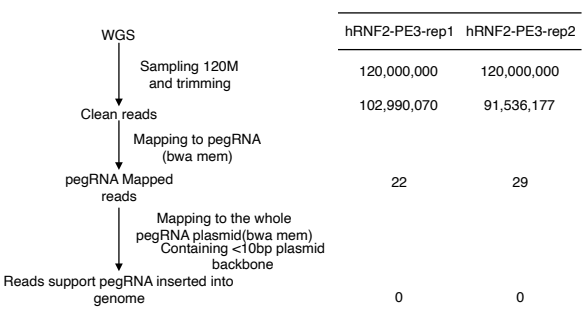

b

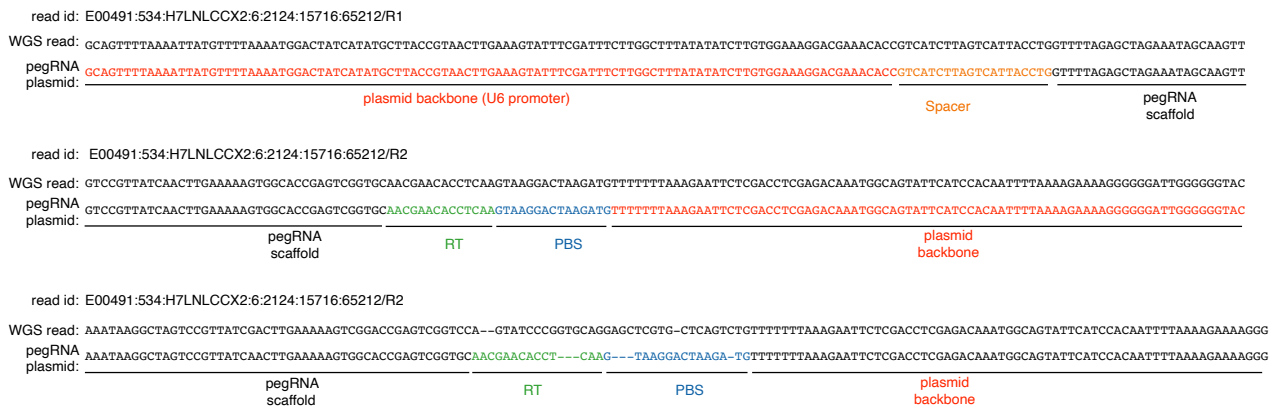

Supplementary Fig. 19. pegRNA backbone sequence was not detected in genome-

\section{wide OT insertions.}

(a) Examining reads that contains potential pegRNA sequence inserted into genome by aligning raw WGS reads of hRNF3-PE3-rep1 and hRNF3-PE3-rep2 to pegRNA sequence. Left: Schematic overview of the pipeline. Right: count of reads pass each step. (b) Examples of pegRNA-containing reads that can be aligned to pegRNA plasmid (vector pU6-pegRNF2) backbone in (a), which are not considered as authentic insertions into genome. 
a
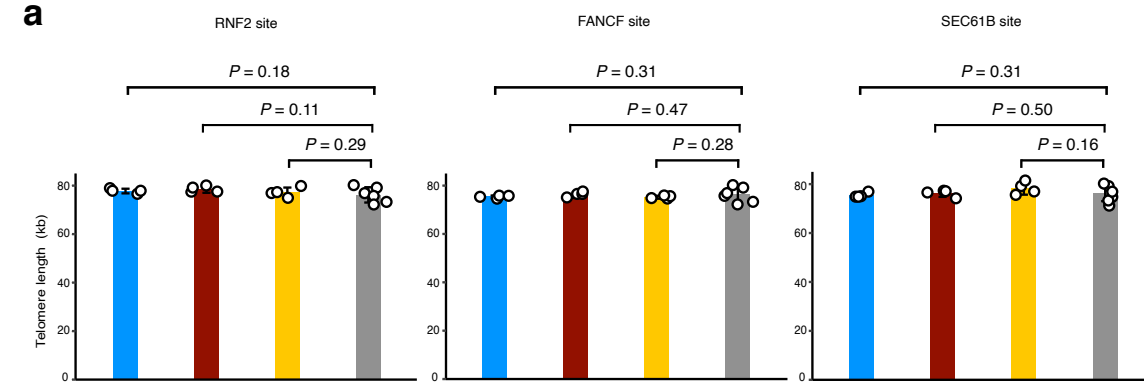

PE3

$P=0.28$

Cas9

EGFP

b

b EMX1 site
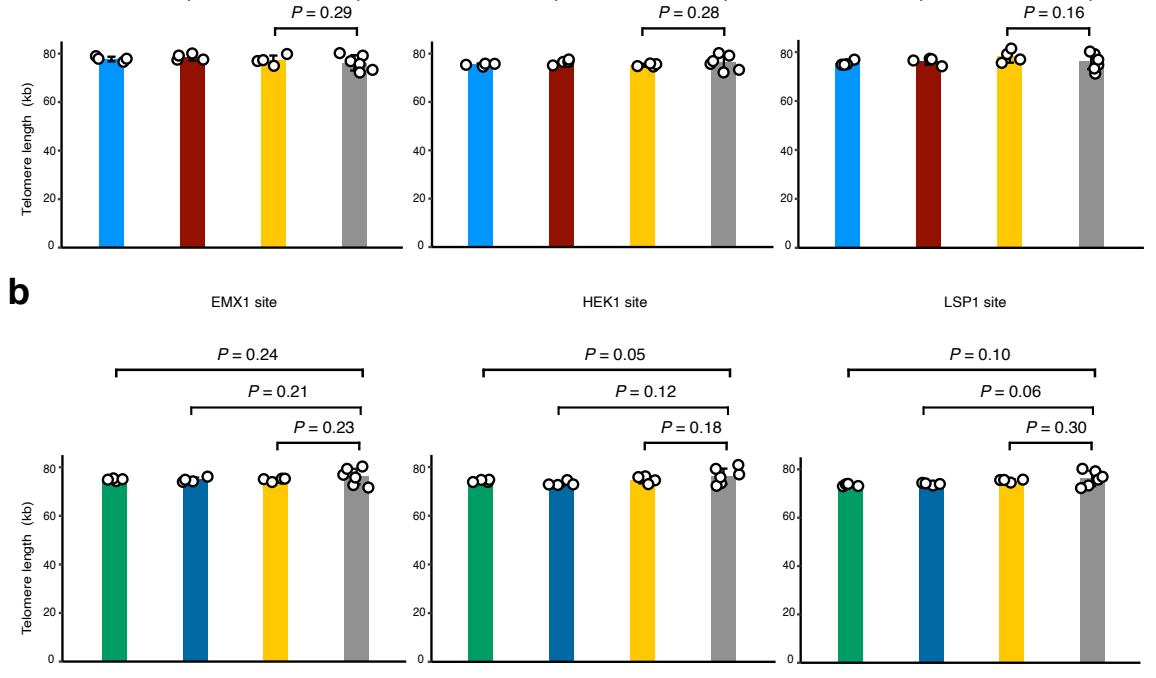

.

c
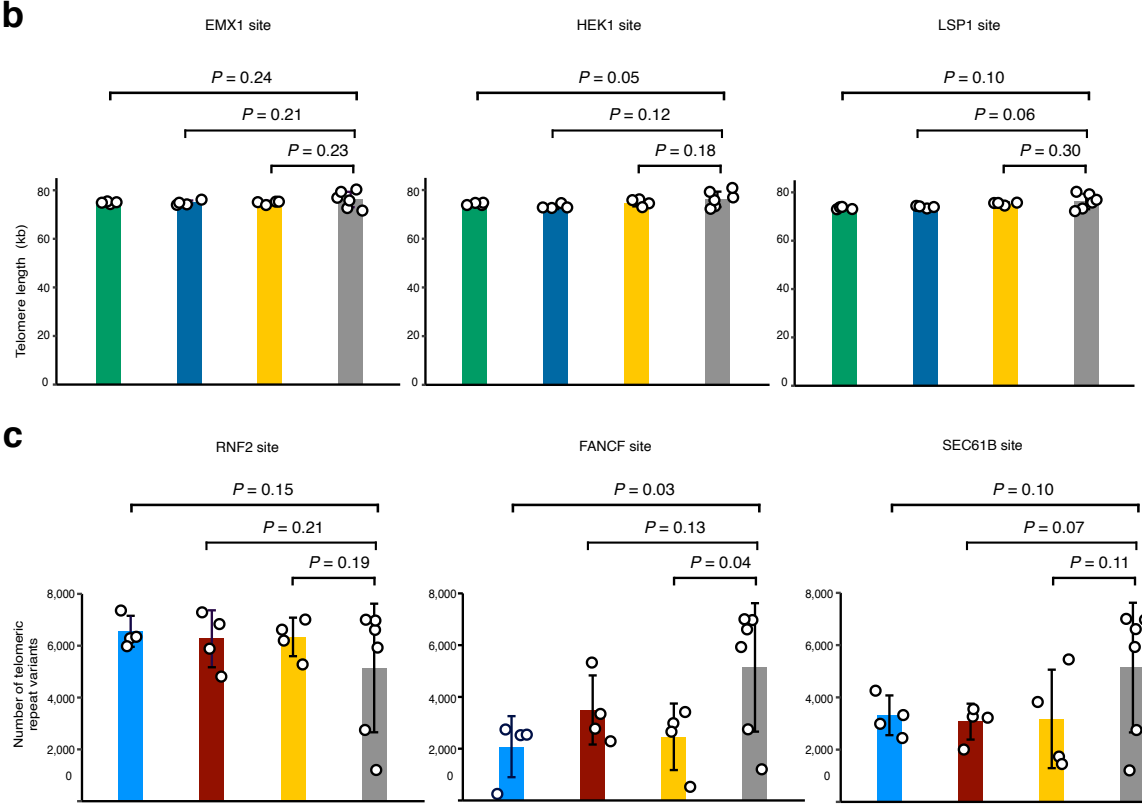

deletion

insertion

Cas9

EGFP

d
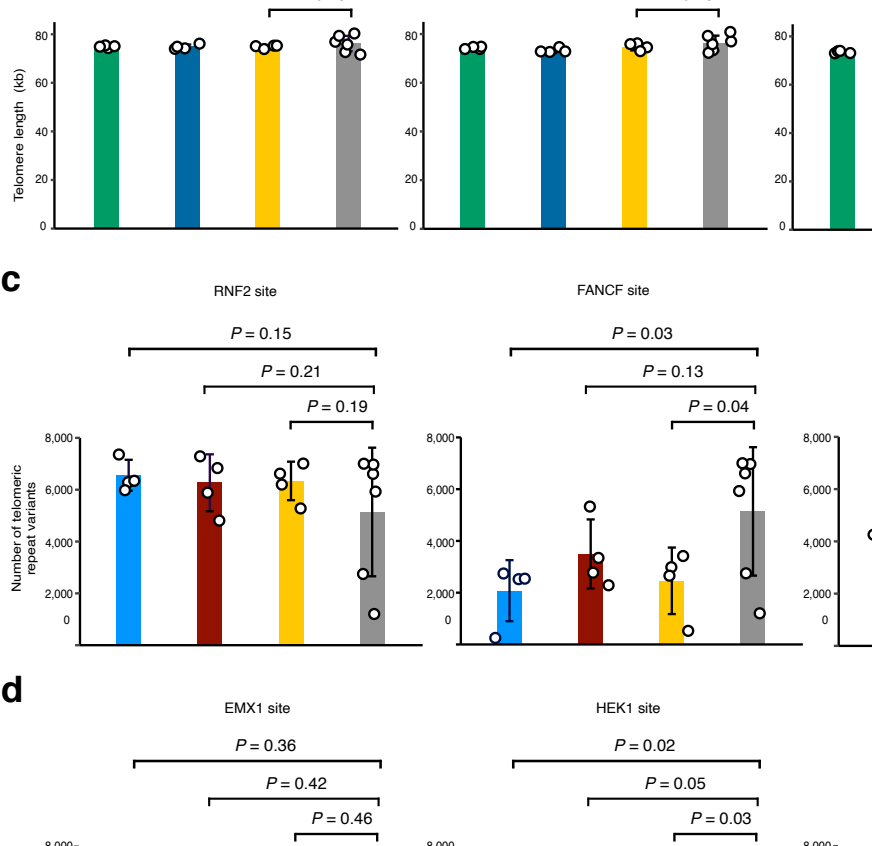

SEC61B site

PE3
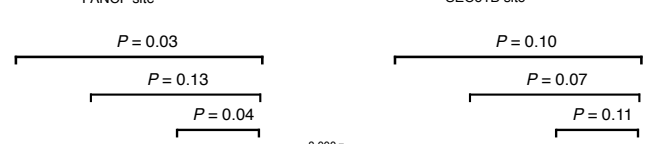

hA3A-BE3

Cas9
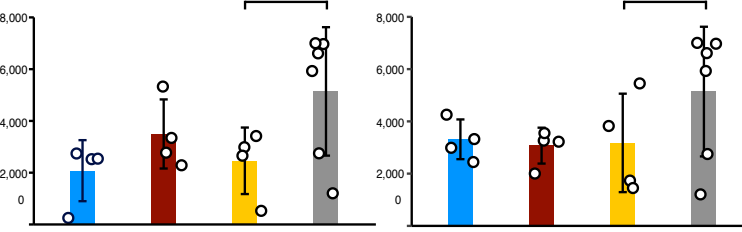

EGFP
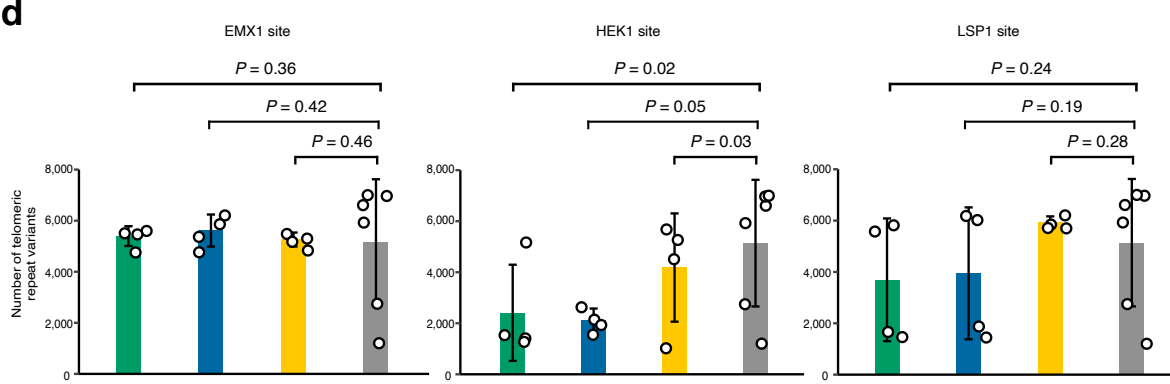

PE3
deletion

PE3

insertion

Cas9

EGFP

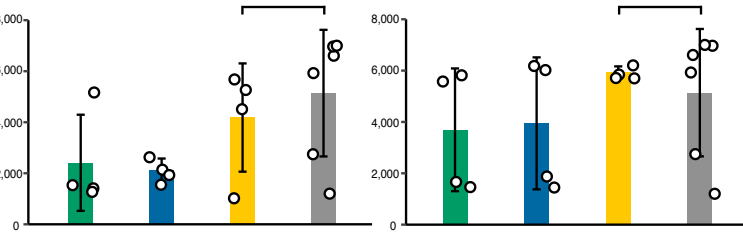

Supplementary Fig. 20. Telomere integrity was not affected by PE3.

(a, b) Telomere lengths in single cell colonies treated by PE3, hA3A-BE3, Cas9 when generating on-target base substitutions (a) or indels (b) were calculated from the WGS data. $(\mathbf{c}, \mathbf{d})$ The number of telomeric repeat variants in single cell colonies treated by PE3 or Cas9 when generating on-target base substitutions (c) or indels (d) 
bioRxiv preprint doi: https://doi.org/10.1101/2021.04.09.439109; this version posted April 9, 2021. The copyright holder for this preprint

(which was not certified by peer review) is the author/funder. All rights reserved. No reuse allowed without permission.

were calculated from the WGS data. Means \pm s.d. were from four independent colonies. 
a
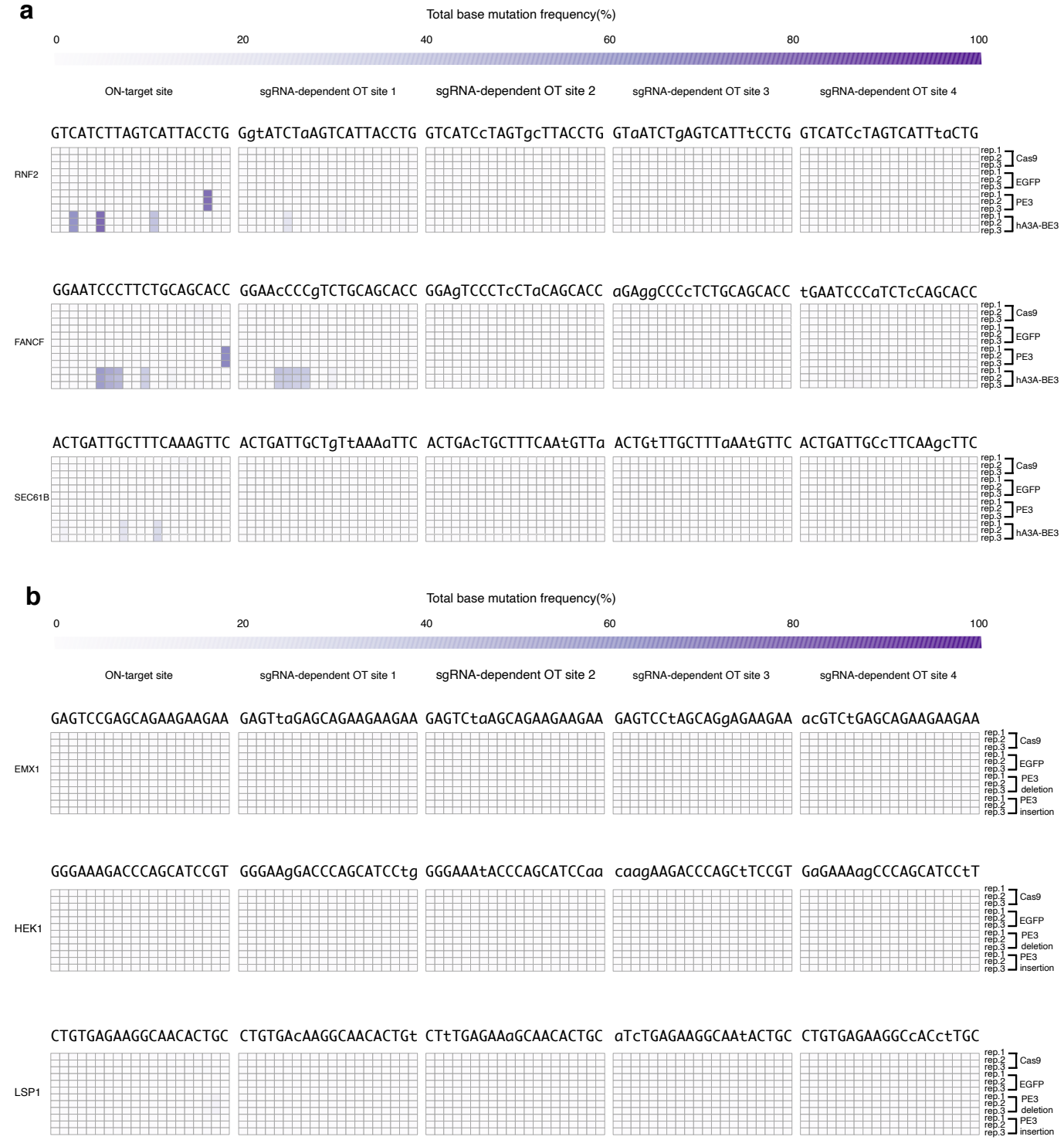

Supplementary Fig. 21. No detectable base substitution induced by PE3 at

gRNA-dependent OT sites in wild-type 293FT cells.

(a) The heatmaps of base substitution frequencies at on-target sites and previously identified or predicted gRNA-dependent OT sites for RNF2, FANCF, SEC61B in WT 293FT cells. (b) The heatmaps of base substitution frequencies at on-target sites and 
bioRxiv preprint doi: https://doi.org/10.1101/2021.04.09.439109; this version posted April 9, 2021. The copyright holder for this preprint

(which was not certified by peer review) is the author/funder. All rights reserved. No reuse allowed without permission.

previously identified or predicted gRNA-dependent OT sites for EMX1, HEK1, LSP

in WT 293FT cells. 


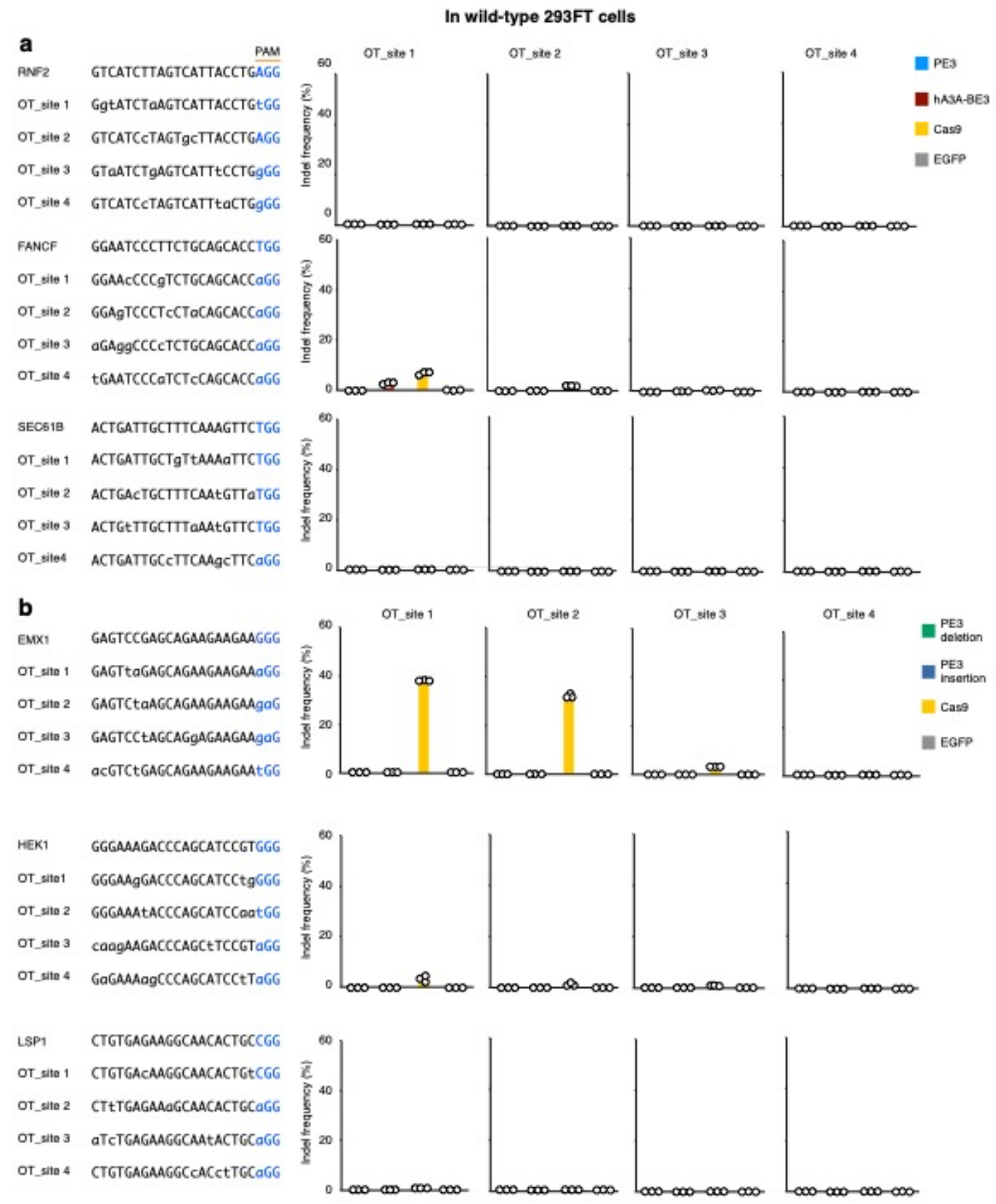

Supplementary Fig. 22. No detectable indel induced by PE3 at gRNA-dependent

\section{OT sites in wild-type 293FT cells.}

(a) The sequences of on-target and previously identified or predicted gRNA-

dependent OT sites for RNF2, FANCF, SEC61B (left) and the indel frequencies at those OT sites (right). (b) The sequences of on-target and previously identified or 
predicted gRNA-dependent OT sites for EMX1, HEK1, LSP (left) and the indel frequencies at those OT sites were shown (right). Means \pm s.d. were from three independent experiments. 


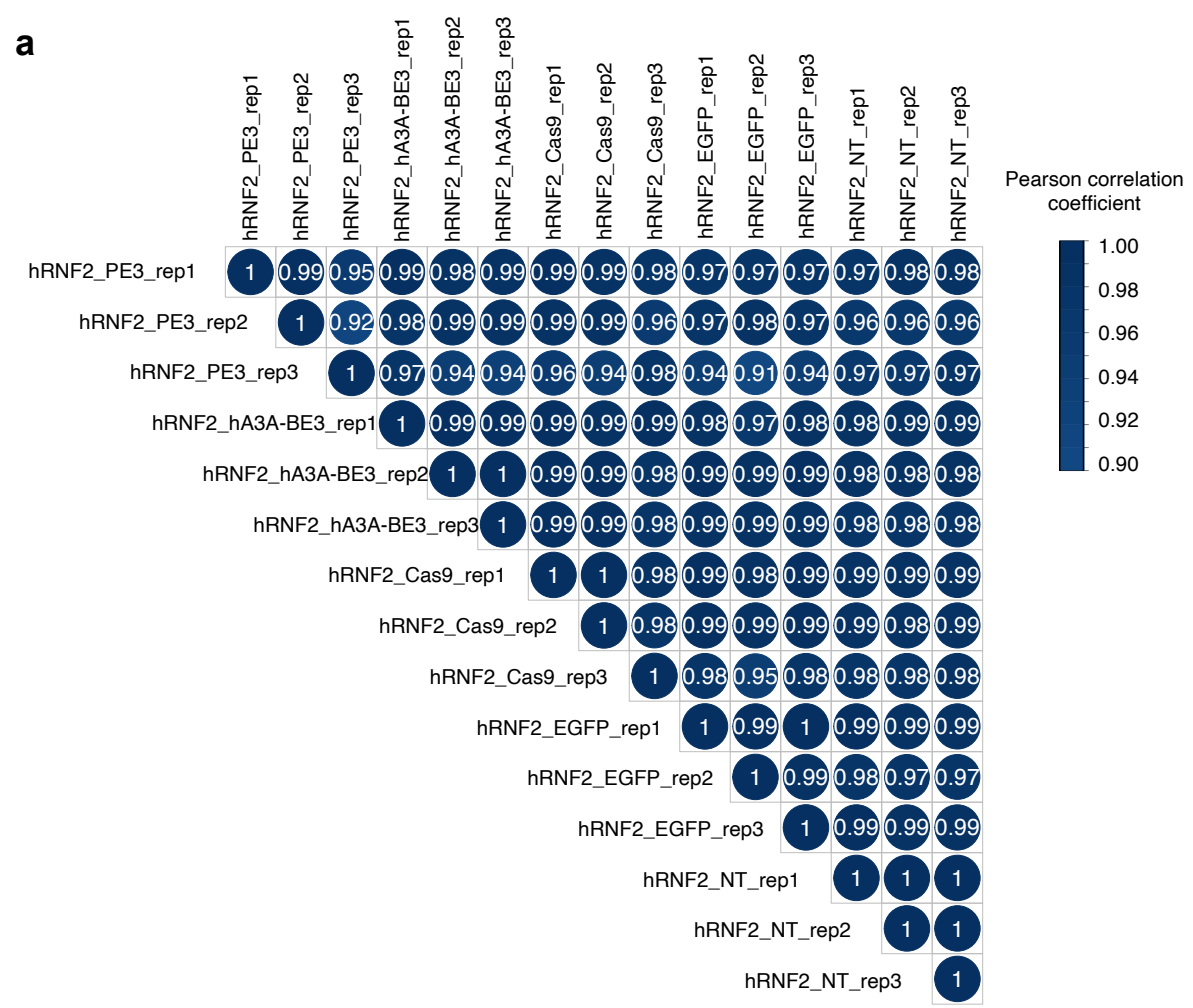

b

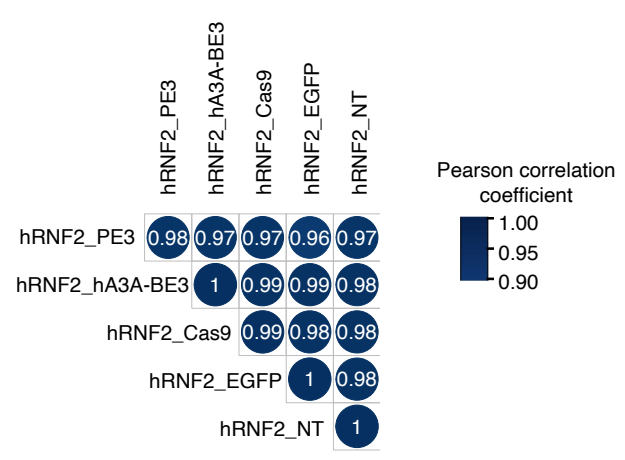

\section{Supplementary Fig. 23. PE3 did not alter gene expression pattern in wild-type}

\section{FT cells.}

(a) Heatmap of Pearson correlations on gene expression level (FPKM, fragments per kilobase of transcript per million mapped fragments) of WT 293FT cells treated with hA3A-BE3, PE3, Cas9, EGFP or left non-treatment (NT). (b) Heatmap of mean

Pearson correlations coefficient across the same treatment on gene expression level 
bioRxiv preprint doi: https://doi.org/10.1101/2021.04.09.439109; this version posted April 9, 2021. The copyright holder for this preprint

(which was not certified by peer review) is the author/funder. All rights reserved. No reuse allowed without permission.

(FPKM) of WT 293FT cells treated with hA3A-BE3, PE3, Cas9, EGFP or left nontreatment (NT). 


\section{SUPPLEMENTARY TABLES}

\section{Supplementary Table 1: Oligos used for plasmid construction.}

\begin{tabular}{|c|c|}
\hline Primer name & Sequence (5' to $\left.3^{\prime}\right)$ \\
\hline hRNF2 FOR & ACCGGTCATCTTAGTCATTACCTG \\
\hline hRNF2_REV & AAACCAGGTAATGACTAAGATGAC \\
\hline hEMX1_FOR & ACCGGAGTCCGAGCAGAAGAAGAA \\
\hline hEMX1_REV & AAACTTCTTCTTCTGCTCGGACTC \\
\hline hFANCF_FOR & ACCGGAATCCCTTCTGCAGCACC \\
\hline hFANCF_REV & AAACGGTGCTGCAGAAGGGATTC \\
\hline hHEK1 FOR & ACCGGGGAAAGACCCAGCATCCGT \\
\hline hHEK1_REV & AAACACGGATGCTGGGTCTTTCCC \\
\hline hLSP1_FOR & ACCGCTGTGAGAAGGCAACACTGC \\
\hline hLSP1_REV & AAACGCAGTGTTGCCTTCTCACAG \\
\hline hSEC61B_FOR & ACCGACTGATTGCTTTCAAAGTTC \\
\hline hSEC61B REV & AAACGAACTTTGAAAGCAATCAGT \\
\hline hA3A_FOR & ACCGCTAGTGGGTGGAATCCGGAA \\
\hline hA3A REV & AAACTTCCGGATTCCACCCACTAG \\
\hline hA3H_FOR & ACCGCTGTACCGAGCTTAATACCT \\
\hline hA3H_REV & AAACAGGTATTAAGCTCGGTACAG \\
\hline +41_nicking-hRNF2_FOR & ACCGTCAACCATTAAGCAAAACAT \\
\hline +41_nicking-hRNF2_REV & AAACATGTTTTGCTTAATGGTTGA \\
\hline+53 nicking-hEMX1_FOR & ACCGGACATCGATGTCCTCCCCAT \\
\hline+53 nicking-hEMX1_REV & AAACATGGGGAGGACATCGATGTC \\
\hline+48 nicking-hFANCF_FOR & ACCGGGGTCCCAGGTGCTGACGT \\
\hline+48 nicking-hFANCF_REV & AAACACGTCAGCACCTGGGACCC \\
\hline +71_nicking-hHEK1_FOR & ACCGAATAGAGGCAAGTATGAGCT \\
\hline +71_nicking-hHEK1_REV & AAACAGCTCATACTTGCCTCTATT \\
\hline -97_nicking-hLSP1_FOR & ACCGAGCATTTGGGGACCTCTTGA \\
\hline -97_nicking-hLSP1_REV & AAACTCAAGAGGTCCCCAAATGCT \\
\hline +46_nicking-hLSP1_FOR & ACCGCCTCTCTGGGCCCTTTGGGT \\
\hline +46_nicking-hLSP1_REV & AAACACCCAAAGGGCCCAGAGAGG \\
\hline +104_nicking-hLSP1_FOR & ACCGGGGCCCCACCCCATCACACA \\
\hline +104_nicking-hLSP1_REV & AAACTGTGTGATGGGGTGGGGCCC \\
\hline -73_nicking-hSEC61B_FOR & ACCGTATTTCAGACACATTAATTA \\
\hline -73_nicking-hSEC61B_REV & AAACTAATTAATGTGTCTGAAATA \\
\hline -29_nicking-hSEC61B_FOR & ACCGTCAGTGCCAAACCATGATTT \\
\hline -29_nicking-hSEC61B_REV & AAACAAATCATGGTTTGGCACTGA \\
\hline+53 nicking-hSEC61B_FOR & ACCGCCCTGCTACATGACGGAGT \\
\hline +53_nicking-hSEC61B_REV & AAACACTCCGTCATGTAGCAGGG \\
\hline+103 nicking-hSEC61B_FOR & ACCGTTACTCCACATTCTGAATGC \\
\hline+103 nicking-hSEC61B_REV & AAACGCATTCAGAATGTGGAGTAA \\
\hline pegRNF2_F & $\begin{array}{l}\text { ATATATCTTGTGGAAAGGACGAAACACCGT } \\
\text { CATCTTAGTCATTACCTGGTTTTAGAGCTAG } \\
\text { AAATAG }\end{array}$ \\
\hline
\end{tabular}




\begin{tabular}{|c|c|}
\hline pegRNF2_R & $\begin{array}{l}\text { GTCTCGAGGTCGAGAATTCTTTAAAAAAAC } \\
\text { ATCTTAGTCATTACTTGAGGTGTTCGTTGCA } \\
\text { CCGACTCGGTGCCA }\end{array}$ \\
\hline pegEMX1_F & $\begin{array}{l}\text { GTGGAAAGGACGAAACACCGGAGTCCGAG } \\
\text { CAGAAGAAGAAGTTTTAGAGCTAGAAATA } \\
\text { G }\end{array}$ \\
\hline pegEMX1-ins-TGC_R & $\begin{array}{l}\text { GTCTCGAGGTCGAGAATTCTTTAAAAAAAT } \\
\text { CCGAGCAGAAGAATGCGAAGGGCTCCCAT } \\
\text { CACGGACCGACTCGGTCCCA }\end{array}$ \\
\hline pegEMX1-del-GGG_R & $\begin{array}{l}\text { TCTCGAGGTCGAGAATTCTTTAAAAAAAAT } \\
\text { CCGAGCAGAAGAAGAACTCCCATCACGCA } \\
\text { CCGACTCGGTGCCA }\end{array}$ \\
\hline pegFANCF_F & $\begin{array}{l}\text { ATATATCTTGTGGAAAGGACGAAACACCGG } \\
\text { AATCCCTTCTGCAGCACCGTTTTAGAGCTA } \\
\text { GAAATAG }\end{array}$ \\
\hline pegFANCF_R & $\begin{array}{l}\text { TCTCGAGGTCGAGAATTCTTTAAAAAAATC } \\
\text { CCTTCTGCAGCACTTGGATCGCTTTTGCACC } \\
\text { GACTCGGTGCCA }\end{array}$ \\
\hline pegHEK1_F & $\begin{array}{l}\text { GTGGAAAGGACGAAACACCGGGAAAGACC } \\
\text { CAGCATCCGTGTTTTAGAGCTAGAAATAGC }\end{array}$ \\
\hline pegHEK1-ins-GTC_R & $\begin{array}{l}\text { TCTCGAGGTCGAGAATTCTTTAAAAAAAAA } \\
\text { AGACCCAGCATCCGTGTCGGGTCGCTGAAA } \\
\text { GCACCGACTCGGTGCCA }\end{array}$ \\
\hline pegHEK1-del-GGG_R & $\begin{array}{l}\text { TCTCGAGGTCGAGAATTCTTTAAAAAAAAA } \\
\text { AGACCCAGCATCCGTTCGCTGAAAGCACCG } \\
\text { ACTCGGTGCCA }\end{array}$ \\
\hline pegLSP1_F & $\begin{array}{l}\text { GTGGAAAGGACGAAACACCGCTGTGAGAA } \\
\text { GGCAACACTGCGTTTTAGAGCTAGAAATAG }\end{array}$ \\
\hline pegLSP1-ins-ATC_R & $\begin{array}{l}\text { TCTCGAGGTCGAGAATTCTTTAAAAAAAAT } \\
\text { GAGAAGGCAACACATCTGCCGGCTGGGGCT } \\
\text { GGCACCGACTCGGTGCCAC }\end{array}$ \\
\hline pegLSP1-del-TGC_R & $\begin{array}{l}\text { TCTCGAGGTCGAGAATTCTTTAAAAAAAAT } \\
\text { GAGAAGGCAACACCGGCTGGGGCTGGCAC } \\
\text { CGACTCGGTGCCAC }\end{array}$ \\
\hline pegSEC61B_F & $\begin{array}{l}\text { ATATATCTTGTGGAAAGGACGAAACACCGA } \\
\text { CTGATTGCTTTCAAAGTTCGTTTTAGAGCTA } \\
\text { GAAATAG }\end{array}$ \\
\hline pegSEC61B_R & $\begin{array}{l}\text { TCTCGAGGTCGAGAATTCTTTAAAAAAAAT } \\
\text { TGCTTTCAAAGTTCTGGTATGAACACGCAC } \\
\text { CGACTCGGTGCCA }\end{array}$ \\
\hline
\end{tabular}


Supplementary Table 2: gRNA target sequences and PCR primer sequences for genomic DNA amplification

\begin{tabular}{|c|c|}
\hline Name & Sequence \\
\hline sgRNF2 & GTCATCTTAGTCATTACCTGAGG \\
\hline hRNF2 G2F & AGGAGGACTTGCCCAACTTT \\
\hline hRNF2 G2R & CTGTGTCAGAACATGCTGGAA \\
\hline sgEMX1 & GAGTCCGAGCAGAAGAAGAAGGG \\
\hline hEMX1_G3F & TCTGTGAATGTTAGACCCATGGG \\
\hline hEMX1_G3R & AGCAGCTGGGAGGCTGCGGTG \\
\hline sgFANCF & GGAATCCCTTCTGCAGCACCTGG \\
\hline hFANCF_G1F & CACAGTATGTCTCTGGCGTT \\
\hline hFANCF_G1R & TCTCTTGGAGTGTCTCCTCA \\
\hline sgHEK1 & GGGAAAGACCCAGCATCCGTGGG \\
\hline hHEK1_G1F & TTGTCGGCAGTAGTGGGAGT \\
\hline hHEK1_G1R & TGGTCTCAAACTCCTGACCT \\
\hline sgLSP1 & CTGTGAGAAGGCAACACTGCCGG \\
\hline hLSP1_G1F & CACTGAACCCTGGGTAGTGA \\
\hline hLSP1 G1R & GACACAGACAGCCAGAAGCT \\
\hline sgSEC61B & ACTGATTGCTTTCAAAGTTCTGG \\
\hline hSEC61B_G1F & CATACCCCACAGATGGAATG \\
\hline hSEC61B_G1R & CCTATCTCATTGCTGCCTGC \\
\hline sghA3A-up & CTAGTGGGTGGAATCCGGAAGGG \\
\hline sghA3H-down & CTGTACCGAGCTTAATACCTGGG \\
\hline hA3A_up_For & GTACCATGAATTGCAAGTGTGT \\
\hline hA3H_dn_Rev & CTGTGTTAGCCAGGATCATCT \\
\hline hRNF2_OTsite-1_G1F & CATCACTGGAGTGAGATGCC \\
\hline hRNF2_OTsite-1_G1R & GGCCGTATAATAGCTAGATGC \\
\hline hRNF2_OTsite-2_G1F & CCCCAGGTGTGCTTTTGAAA \\
\hline hRNF2_OTsite-2_G1R & AAAGGCGGTCCCACAACGACAT \\
\hline hRNF2_OTsite-3_G1F & GGCTCCAACCAAGTTAAATCAGG \\
\hline hRNF2_OTsite-3_G1R & CCTCCTGGATACTGATATAC \\
\hline hRNF2_OTsite-4_G1F & AGGTGACATGTGAGCTGCTG \\
\hline hRNF2_OTsite-4_G1R & AACTTTCCCACGGAGCACAG \\
\hline hEMX1_OTsite-1_G1F & TCCCTGTCTTCCAGGAATGT \\
\hline hEMX1_OTsite-1_G1R & CCTTCAACTCATGACCAGCTT \\
\hline hEMX1_OTsite-2_G1F & CCACATCTTCACTAATACGGT \\
\hline hEMX1_OTsite-2_G1R & GAATATTGAGTCATGTGGCAGT \\
\hline hEMX1_OTsite-3_G1F & TCTTGGCTTCCTGTCACCTC \\
\hline hEMX1_OTsite-3_G1R & CACTGCAGCCTCAAACTCCT \\
\hline hEMX1_OTsite-4_G1F & GTCAACAAAGCCAGCCTCAT \\
\hline hEMX1_OTsite-4_G1R & TTTCATAAGTCAGTGCATCC \\
\hline hFANCF_OTsite-1_G1F & ACCACCATGCACCTTAAAGC \\
\hline hFANCF_OTsite-1_G1R & CGGTTCAGGATAATGCCACT \\
\hline hFANCF_OTsite-2_G1F & CTGAGGTGCAAGAAGTAATGGT \\
\hline hFANCF_OTsite-2_G1R & AAGGTGAAGGCAGTAGAGAGA \\
\hline hFANCF_OTsite-3_G2F & GGGGTCCTTCTTTGGACCTA \\
\hline hFANCF_OTsite-3_G2R & CAACGTTCGAGGCAATGTC \\
\hline
\end{tabular}




\begin{tabular}{|c|c|}
\hline hFANCF_OTsite-4_G1F & AAGGCCAGATGAGTGTAGCC \\
\hline hFANCF_OTsite-4_G1R & CTCTGGCATCCTGACAAACA \\
\hline hHEK1_OTsite-1_G1F & AATCTATAGCGCTGAGAAGC \\
\hline hHEK1_OTsite-1_G1R & AGATCTGCACCCAGACCTGT \\
\hline hHEK1_OTsite-2_G1F & TTCAAGGGAGCTAACGAGTT \\
\hline hHEK1_OTsite-2_G1R & AGGGCACAGGAATACATCAT \\
\hline hHEK1_OTsite-3_G1F & AAACCATCTCCGACAGCCTT \\
\hline hHEK1_OTsite-3_G1R & GGGTGTGCAAAACCGAAGAT \\
\hline hHEK1_OTsite-4_G2F & ACCGTGATTGTGACTGTAAA \\
\hline hHEK1_OTsite-4_G2R & GAAGGTCAGCAAACAGAGAA \\
\hline hLSP1_OTsite-1_G1F & TCATGCCACCCAGAATCCAG \\
\hline hLSP1_OTsite-1_G1R & TACCTACCTGTGCCCTTTAA \\
\hline hLSP1 OTsite-2 G1F & AAACCTGTAACTTGAGAACC \\
\hline hLSP1_OTsite-2_G1R & TTTTAGCACTGCATGGTGTT \\
\hline hLSP1_OTsite-3_G2F & GTCAGAAGTTTGAAATCGCT \\
\hline hLSP1_OTsite-3_G2R & CAGATCCTGGGGTTTCTCAT \\
\hline hLSP1_OTsite-4_G2F & TATAGGACTTGGGCTTATGT \\
\hline hLSP1_OTsite-4_G2R & CTTTTGACATATAGGGCCTA \\
\hline hSEC61B_OTsite-1_G2F & ATCTACTAGCCCACTGCTGG \\
\hline hSEC61B_OTsite-1_G2R & CAGGAGAATGGCGTGAACTT \\
\hline hSEC61B_OTsite-2_G2F & ATCGGCTCATACTGCATATA \\
\hline hSEC61B_OTsite-2_G2R & AGACCTGTCACATAAGGTCA \\
\hline hSEC61B OTsite-3 G2F & GCTCCAATGCCACGTGTTAA \\
\hline hSEC61B_OTsite-3_G2R & CTTAATTTGGGCATTCCTGG \\
\hline hSEC61B OTsite-4_G1F & ACGAGAGACTGTAACTTTCT \\
\hline hSEC61B OTsite-4 G1R & AATTACACTGCTTTCTCAGC \\
\hline hANK2_G1F & GAGGCATCATTGCTTAGAAC \\
\hline hANK2_G1R & AGATGGTCCCCTTCTTTCTG \\
\hline hCAPN10_G2F & TGCAGCTACGGGGTTCTTGG \\
\hline hCAPN10_G2R & GGCCTCCTGTCCTCTCCAAA \\
\hline hCELSR2_G1F & TTTGTGCCATGTTCTCTCCA \\
\hline hCELSR2_G1R & CTCAGCTGTTCCTAAAGGGA \\
\hline hEEPD1_ $\bar{G} 2 \mathrm{~F}$ & TGTGGTGAGAGAAGGCCTCA \\
\hline hEEPD1_G2R & TCTGTGGCATCCAGAGATAA \\
\hline hHSPG2_G2F & TCCAGCACTCAGTTTTCTGG \\
\hline hHSPG2_G2R & GATCAGGAGCTCATCCAGGT \\
\hline hNIF3L1_G1F & CCTTACGAGCGATAGAACAT \\
\hline hNIF3L1_G1R & CTTTTGTGTTTCCTCATTCC \\
\hline hRASSF1_G2F & AGCAGTAATTTGTGCATACC \\
\hline hRASSF1_G2R & AAGCTCAGGGCCTTGTCACT \\
\hline hTG_G1F & GGTGAATGTTGGCCAGTTTA \\
\hline hTG_G1R & CTCTTTGCCCCAGGAATTCA \\
\hline hTMEM181_G1F & TAGGCAATTCAGCCAGGGCT \\
\hline hTMEM181_G1R & CACCATGCCCAGCTAAATTT \\
\hline hVAV1_G1F & TCTGTTGGGACAGCTTGTCT \\
\hline hVAV1_G1R & GAGCATAAGTTTAAGGTCAC \\
\hline
\end{tabular}




\section{Supplementary Table 3: Calculation of on-target base substitutions. Base}

substitutions were determined by deep sequencing and base substitution frequencies at the positions in gRNA-targeted genomic loci were calculated for indicated conditions. Read counts for all four types of bases are listed.

Supplementary Table 4: Calculation of on-target indels. Indels were determined by deep sequencing and indel frequencies at the examined gRNA-targeted genomic loci were calculated for indicated conditions. Counts of indel-containing reads and total mapped reads are listed.

\section{Supplementary Table 5: Calculation of gRNA-dependent OT base substitutions}

and indels. Base substitutions and indels were determined by deep sequencing and total base substitution frequencies and indel frequencies at the examined gRNAdependent OT genomic loci were calculated for indicated conditions. Read counts for all four types of bases and counts of indel-containing reads and total mapped reads are listed.

Supplementary Table 6: Genome-wide base substitutions and indels. Genomewide base substitutions and indels were determined by whole-genome sequencing for indicated single colonies.

\section{Supplementary Table 7: Calculation of telomere lengths and the numbers of} telomeric repeat variants. Telomere lengths and the numbers of telomeric repeat variants were determined by whole-genome sequencing for indicated single colonies. 
Supplementary Table 8: Transciptome-wide mutations. Transcriptome-wide mutations were determined by whole-transcriptome sequencing for indicated conditions . 